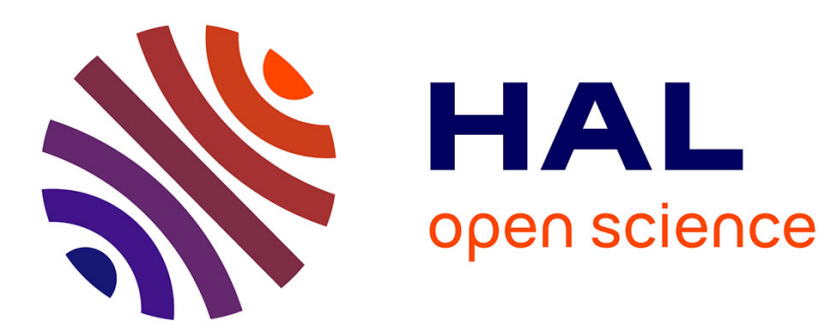

\title{
Stimuli-Responsive Toughening of Hydrogels
}

Xinxing Lin, Xiaolin Wang, Liangpeng Zeng, Zi Liang Wu, Hui Guo,

Dominique Hourdet

\section{To cite this version:}

Xinxing Lin, Xiaolin Wang, Liangpeng Zeng, Zi Liang Wu, Hui Guo, et al.. Stimuli-Responsive Toughening of Hydrogels. Chemistry of Materials, 2021, 10.1021/acs.chemmater.1c01019 . hal03346113

\section{HAL Id: hal-03346113 \\ https://hal-espci.archives-ouvertes.fr/hal-03346113}

Submitted on 16 Sep 2021

HAL is a multi-disciplinary open access archive for the deposit and dissemination of scientific research documents, whether they are published or not. The documents may come from teaching and research institutions in France or abroad, or from public or private research centers.
L'archive ouverte pluridisciplinaire HAL, est destinée au dépôt et à la diffusion de documents scientifiques de niveau recherche, publiés ou non, émanant des établissements d'enseignement et de recherche français ou étrangers, des laboratoires publics ou privés. 
1 Stimuli-Responsive Toughening of Hydrogels

2

4 a. School of Chemical Engineering and Technology, Sun Yat-sen University, Zhuhai, 5 519082, China.

6

9 Functionalization, Department of Polymer Science and Engineering, Zhejiang University,

10 Hangzhou 310027, China.

11

12 University, CNRS, Paris F-75005, France. 
14 ABSTRACT

15 Materials with stimuli-switchable properties are widespread in nature. Through

16 biomimicry, stimuli-responsive hydrogels have received increasing research in recent

17 years. In particular, hydrogels with stimuli-tunable mechanical properties provide an

18 important platform for designing new materials with specific applications such as 3D

19 printing, soft robot, and tunable adhesion. Herein, the state-of-the-art of hydrogels with

20 stimuli-responsive toughness is reviewed in detail. Characteristic reinforcement

21 mechanisms are fully discussed first, followed by systematical demonstrations of various

22 smart hydrogels responding to various stimuli. In particular, special attention is paid to

23 thermal, light, $\mathrm{pH}$, and salt triggers because of their broad applications and easy

24 implementation. Furthermore, the applications of these smart hydrogels are included. In

25 addition to the overview of recent advances, we finally summarize the critical challenges

26 of current discoveries and make an outlook for the following work, which we anticipate to

27 lead to substantial progress of these responsive materials in the future. 
30 Living materials consist of various smart components whose performance can be finely

31 tuned by stimuli. For instance, muscles reversibly stiffen or soften in response to neutral

32 signals, chameleons hold the ability to change color in response to several environmental

33 triggers, and Mimosa Pudica leaves close automatically under mechanical disturbances.

34 Learning from nature, bio-inspired materials with stimuli-responsiveness have attracted

35 considerable attention during the last few decades. ${ }^{1}$ In particular, stimuli-responsive

36 hydrogels, which are often termed as smart hydrogels, have been intensively investigated

37 as they automatically respond to environmental changes. ${ }^{2-3}$ Bearing high level of water

38 contents, hydrogels serve as ideal candidates for bio-engineering applications as they share

39 versatile similarities with human tissues. ${ }^{4-5}$ Moreover, their ability to be programmed

40 concerning one or various given stimuli considerably broadens their application

41 perspectives. Typical stimuli involve temperature, $\mathrm{pH}$, humidity, light, specific ions or

42 molecules, electrical field, magnetic field, solvent, and ionic strength. Therefore, these

43 smart hydrogels demonstrate great potential for developing specific applications, from

44 targeted drug delivery ${ }^{6}$ to precisely controlled actuators, ${ }^{7-8}$ from artificial muscles ${ }^{9}$ to self-

45 healable tissue, ${ }^{10}$ and from photochromic windows ${ }^{11}$ to magnetic sensors. ${ }^{12}$

46 When subjected to external stimuli, the response of hydrogels is transmitted from the

47 microscopic level to the macroscopic scale. Their optical transparency is easily tunable,

48 their size and shape are readily controllable, and their hydrophilicity is finely adjustable. ${ }^{13}$ 
49 Apart from these features, smart hydrogels can also demonstrate switchable mechanical

50 performance, with dramatic modifications of their rigidity, extensibility, and toughness.

51 Therefore, these hydrogels are able to adapt to complex environments and can be used in

52 relevant applications where stimuli-tunable soft materials are required. For example, a soft

53 robot needs the mechanical responsiveness of its components in order to accomplish a

54 complex movement. ${ }^{14}$

55 While stimuli-responsive hydrogels have already been adequately addressed and fully

56 summarized based on their tunable size, ${ }^{15}$ color, ${ }^{16}$ and even electro-conductivity, ${ }^{2}$ the

57 control of their mechanical properties by environmental stimuli has been much less

58 investigated and rationalized. To fill this gap, recent advances in the field of three-

59 dimensional hydrogels with stimuli-responsive toughness are highlighted and summarized

60 in this review (Figure 1). Associating polymer solutions that undergo sol-gel transition and

61 form transient unstable weak networks are excluded from the discussion. In the first part,

62 the different mechanisms involved in the viscoelastic response of hydrogels are carefully

63 discussed before describing the main stimuli used to modify their mechanical performances.

64 Special attention is given to thermal, light, $\mathrm{pH}$, and salt responsive hydrogels. The

65 responsive mechanical properties of hydrogels are explored and both the advantages and

66 weaknesses of these smart systems are discussed. Finally, this review provides concluding

67 remarks over the recent advances and challenges in this field with some guiding ideas

68 which could inspired new findings. 

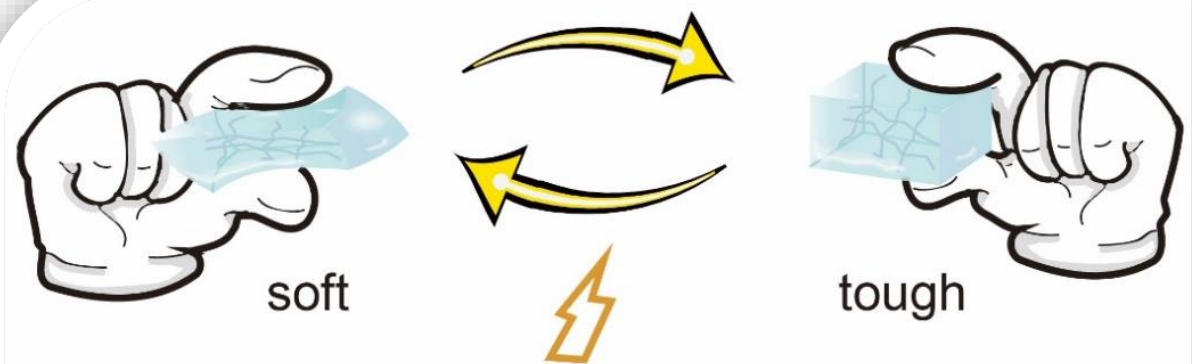

\section{Environmental stimuli $\wedge$}

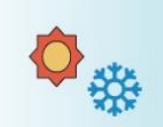

temperature

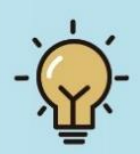

light

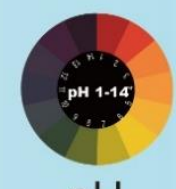

$\mathrm{pH}$

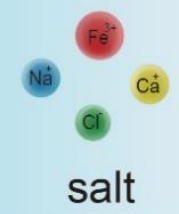

Figure 1. Schematic illustration of hydrogels with stimuli-responsive mechanical performance.

\section{Mechanisms of hydrogels with responsive mechanical performance}

73 Before discussing the stimuli-responsiveness, it is important to briefly recall some basic

74 elements relating to the viscoelastic properties of hydrogels, typically the elastic and

75 dissipative behaviors. Hydrogels are soft materials composed of crosslinked polymer

76 chains typically swollen with water. While absorbing a large amount of solvent inside the

77 three-dimensional network with osmotic pressure contribution, hydrogels still maintain

78 solid-state with certain levels of mechanical performance. The swelling and elasticity of

79 hydrogels are directly related to the crosslink density of the network, its polymer volume

80 fraction as well as the interactions between solvent and polymer. According to the classical 
81 theory of rubber elasticity, ${ }^{17-18}$ the shear modulus $G$ of swollen hydrogel having no

82 permanent (trapped) entanglements can be described by the relation below,

$$
G \cong k T v_{e, \phi}=\frac{R T \rho_{0} \phi_{0}^{a} \phi^{b}}{M_{0} N}
$$

84 with $v_{e, \phi}$ the number density of elastically active chains at the polymer volume fraction $\phi$,

$85 \mathrm{k}$ the Boltzmann constant, $R$ the gas constant, $T$ the temperature, $\phi_{0}$ the polymer volume

86 fraction in the preparation state, $\rho_{0}$ the polymer density, $M_{0}$ the molar mass of the monomer,

87 and $N$ the number of monomers per elastic strand. In equation (1), $a$ and $b$ are scaling

88 exponents which depend on the quality of the solvent $(a=2 / 3, b=1 / 3$ in $\theta$ conditions and

$89 a=5 / 12, b=7 / 12$ in a good solvent), with $G \sim \phi_{0}$ for gels in the preparation state $\left(\phi=\phi_{0}\right)$.

90 From equation (1), we can see that the polymer volume fraction, the quality of solvent, and

91 the molar mass between crosslinks determine the elastic modulus for a given hydrogel.

92 This concentration dependence is valid for chemical networks having no permanent

93 (trapped) entanglements which means that $\mathrm{N}$ is shorter than the length of an entanglement

94 strand at the preparation concentration $\mathrm{N}_{\mathrm{e}}\left(\phi_{0}\right)$. In the opposite situation, $\mathrm{N}_{\mathrm{e}}\left(\phi_{0}\right)<\mathrm{N}$, the

95 elastic properties are dominated by entanglements and $G \sim R T \phi_{0}^{c} \phi^{d}(c=2, d=1 / 3$ in $\theta$

96 conditions and $c=5 / 3, d=7 / 12$ in a good solvent); with $G \sim \phi_{0}^{2.3}$ for gels in the preparation

97 state. ${ }^{18}$ All these equations derived from linear elasticity, assuming Gaussian statistics for

98 polymer chains, hold at low deformation. This is no longer the case when the chains are

99 subjected to larger deformations where more sophisticated statistical approaches may be 
100 used, like Langevin statistics ${ }^{19}$ to model the nonlinear rubber elasticity. This is the case for 101 highly swollen polyelectrolyte networks which become stiffer ( $G$ increases) when the 102 chains are stretched with the swelling ( $v_{\mathrm{e}, \phi}$ decreases $) .{ }^{20}$

103 While the stiffness of swollen polymer networks is something relatively well understood 104 and quite easily controlled, hydrogels remain fragile materials. Indeed, they are 105 characterized by low mechanical properties in terms of extensibility and tenacity which are 106 currently a hot topic in the scientific community of hydrogels. Experimentally, the 107 measured strain and stress at break of material correspond to the point where a crack 108 propagates through the sample. Therefore, we can assume, as proposed by Maugis and 109 Barquins, ${ }^{21}$ that the interfacial fracture energy $\Gamma(v)$ can be empirically written as:

$$
\Gamma(v)=\Gamma_{0}\left(1+f\left(a_{T} v\right)\right)
$$

111 where $f\left(a_{T} v\right)$ is a velocity-dependent dissipative factor with $v$ the crack velocity and $112 a_{T}$ the time-temperature superposition shift factor; $\Gamma_{0}$ is the threshold fracture energy for 113 vanishing crack velocities, mostly related to the chemistry of the interface. Based on the 114 molecular theory for $\Gamma_{0}$ developed by Lake et $a l,{ }^{22}$ when any of the main chain bonds break, 115 the total bond energy of each bond of the stretched chain is irreversibly lost. Therefore, the 116 minimum energy necessary to break the chain is proportional to the length of that chain, 117 i.e., to the number of $\mathrm{C}-\mathrm{C}$ bonds comprising that $N$-chain. Assuming that only the strands 118 crossing the fracture plane will break for a homogeneously crosslinked hydrogel, $\Gamma_{0}$ can be 
119 written as: $:^{22-23}$

$$
\Gamma_{0}=N U_{b} \Sigma \approx U_{b} v_{e, \varphi=1} \phi a N^{\frac{3}{2}} \approx \frac{U_{b} a \rho_{0} \phi}{M_{0}} N^{\frac{1}{2}}
$$

121 where $\Sigma$ is the areal density of strands crossing the interface, $U_{b}$ is the bond energy of a C-

$122 \mathrm{C}$ bond $(\sim 350 \mathrm{~kJ} / \mathrm{mol}), v_{e, \varphi}=1$ the number density of elastically active chains in the melt

123 state and $a$ is the size of the monomer. From this equation, it turns out that the fracture

124 energy of hydrogels is also related to their polymer volume fraction and crosslinking

125 density, though the dependence is distinct from that on elastic shear modulus. Indeed, the

126 crosslinking density, which is directly related to reciprocal $N$, is positively related to $G$ but

127 negatively contributes to $\Gamma$. Consequently, there is a trade-off between rigidity and

128 toughness. In contrast, both $G$ and $\Gamma$ demonstrate a consistent relationship to $\phi$, which

129 means that both rigidity and toughness decrease when the gel swells; i.e. $\phi$ decreases.

130 Based on equation (2), the velocity-dependent dissipative factor $f\left(a_{T} v\right)$ also plays an

131 important role in determining hydrogels' toughness. This factor, which considers the

132 mechanical dissipation in the bulk, is related to the crack velocity $v$ and the time-

133 temperature superposition shift factor $a_{T}$. Along with the crack propagation velocity, the

134 rise of molecular friction and noncovalent interactions between polymer chains increase

135 dissipation processes and these effects have been clearly shown to significantly increase

136 the fracture energy of soft materials. ${ }^{24-25}$ For water-swollen hydrogels, which are generally

137 characterized by a low polymer volume fraction and a weak dissipative factor, physical 
138 interactions ${ }^{26-27}$ that can reversibly break under stretch can significantly enhance the 139 hydrogels toughness. Moreover, the plasticity of the gel, which can be implemented by 140 incorporating stiffer domains that can only be deformed above a certain stress level, can 141 also greatly contribute to the hydrogel strengthening. ${ }^{28-29}$

142 Considering the above theories, the mechanical performances of hydrogels strongly depend 143 on the crosslink density, the volume fraction, and the polymer chain interactions. By tuning 144 these factors with external triggers, stimuli-responsive hydrogels can toughen or soften on 145 demand. As shown in Figure 2, the typical mechanisms involve noncovalent interactions, 146 formation or scission of covalent bonds, change in water content, phase separation, etc.

a)

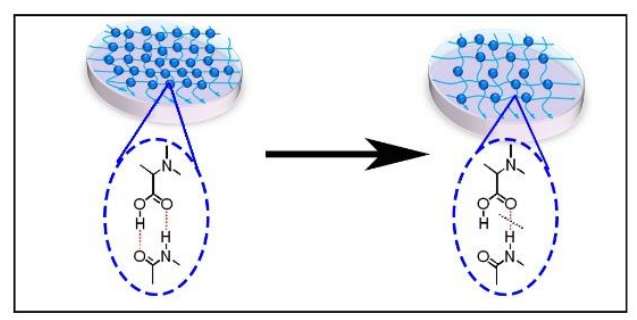

c)

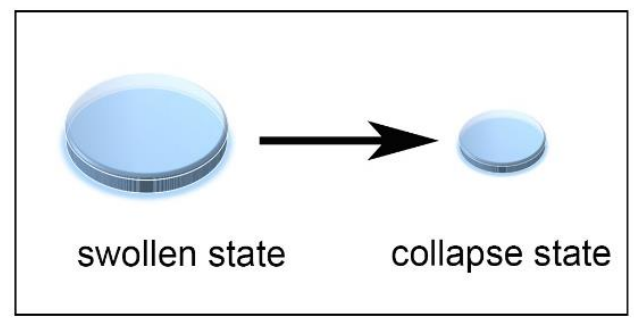

b)

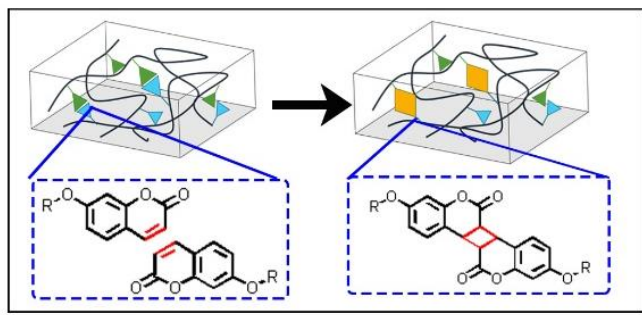

d)

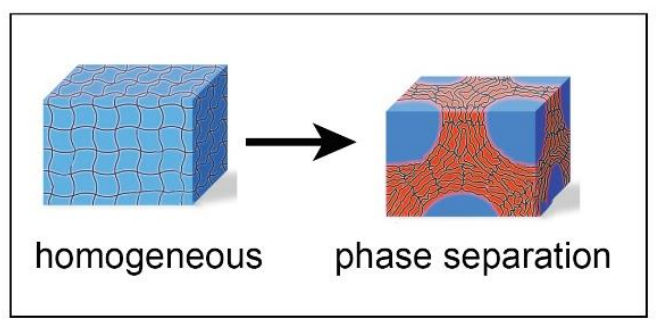

Figure 2. Mechanisms of hydrogels with responsive toughness. a) Noncovalent interactions. b) Formation or scission of covalent bonds. c) Volume change. d) Phase separation. Reproduced with permission. ${ }^{30}$ Copyright 2020 Wiley-VCH Verlag GmbH \& Co. KGaA. 
$152 \quad 1.1$ Influence of noncovalent interactions

153 Generally, stimuli-responsive hydrogels develop various noncovalent interactions within 154 their three-dimensional polymer networks. ${ }^{24,31}$ These noncovalent interactions includes 155 hydrophobic interactions, ${ }^{32}$ hydrogen-bonds, ${ }^{33}$ dipole-dipole interactions, ${ }^{34}$ coordinate 156 bonds, ${ }^{35}$ etc. These interactions may serve as dynamic bonds under stretch, thus increasing 157 the dissipative factor and then toughening the hydrogels. When subjected to environmental 158 signals, the strength of these physical interactions can be reversibly/irreversibly influenced.

159 For example, hydrogen bonds are generally temperature-dependent, ${ }^{36}$ the strength of 160 coordinate bonds is often sensitive to environmental $\mathrm{pH}$ conditions ${ }^{37-38}$ and the association 161 between anions and cations is likely to be screened by the addition of salts. ${ }^{39}$ Therefore, 162 the application of environmental stimuli can easily lead to responsive stiffening or 163 weakening of hydrogels.

$164 \quad 1.2$ Formation or scission of covalent bonds

165 When experiencing environmental variations, new covalent bonds may form or/and 166 existing covalent bonds may break. Along with the formation or scission of covalent bonds, 167 the effective crosslinking degree of hydrogels is affected and consequently its mechanical 168 properties. This is somewhat more ubiquitous in light-responsive hydrogels, as the high 169 energy of light is able to readily influence chemical bonds, including the cleavage, ${ }^{40}$ 170 addition, ${ }^{41}$ exchange, ${ }^{42-43}$ and isomerization. ${ }^{44-45}$ The shape and toughness of light-sensitive 
171 hydrogels can be modulated by adjusting wavelength or time of irradiation. ${ }^{41}$

$172 \quad 1.3$ Volume change

173 Both elastic and fracture behavior of hydrogel are highly affected by its water content.

174 According to the theories of rubber elasticity and Lake-Thomas ${ }^{46}$ discussed previously, 175 both rigidity $(G)$ and toughness $(\Gamma)$ of hydrogels increase with the polymer volume fraction

$176(\phi)$ and then decrease with the swelling of the gel $\left(Q=1 / \phi=V_{s w} / V_{d r y}\right)$, with $Q$ the 177 swelling ratio and $V_{s w}, V_{d r y}$ the swollen and dry volumes of the gel. External stimuli may 178 then induce a variation of the osmotic pressure within the polymer network, giving rise to 179 water diffusion inside or outside the gel which will consequently affect its mechanical 180 properties. At the same time, large volume contractions can also induce stronger polymer 181 interactions ${ }^{47}$ thus leading to further reinforcement of the hydrogels.

182 1.4 Phase separation

183 Upon stimuli changing the environmental conditions, water may shift from a good solvent 184 to a poor solvent for the polymer network. In such a case, the gels tend to become opaque 185 due to phase separation giving rise to an alternation of dense and sparse polymer regions 186 forming discontinuous or bicontinuous domains at the submicron scale. ${ }^{48-49}$ While the rich 187 polymer phase forms a load-bearing scaffold percolating through the whole network in 188 response to the stimulus, the mechanical performances of the gel are simultaneously 
189 modulated. For instance, Shibayama et al. have detailed the change of viscoelastic 190 properties of thermo-responsive hydrogels upon phase separation. ${ }^{50}$ Compared to other 191 aforementioned mechanisms, the phase separation process has a much greater impact on 192 the reinforcement of hydrogels. When a hydrophilic polyacrylamide hydrogel is transferred 193 from water to a $N, N$-dimethylformamide (DMF)/water mixture, the gel undergoes rapid 194 phase separation and demonstrates a strong improvement in stiffness, toughness, with self195 healing properties and an increased elastic modulus over more than 4 decades. ${ }^{51}$ A similar 196 behavior was reported with the phase separation of $N, N$-dimethylacrylamide hydrogel 197 induced by inorganic additives with an abrupt change of mechanical properties around the 198 transition point. ${ }^{52}$

199 It is worthwhile mentioning that more than one mechanism may play a synergistic effect 200 in the responsive regulation of the mechanical performances of smart hydrogels. Taking 201 for instance thermo-responsive $\operatorname{poly}(N$-isopropylacrylamide) hydrogel, the material is 202 remarkably strengthened when heated above the phase transition temperature with the 203 synergistic contribution of enhanced physical interactions, shrinkage, and phase 204 separation. ${ }^{53}$ Although the above mechanisms generally go hand in hand, it is important to 205 stress that the phase separation phenomenon largely dominates the others. It can even lead 206 to a mechanical reinforcement of the gel even when the latter swells during the phase 207 separation, as recently shown in the case of organogels immersed in water. ${ }^{54}$ 
208 Various physical or chemical stimuli may induce toughening or weakening of hydrogels

209 according to the mechanisms discussed above. Within the field of responsive hydrogels,

210 thermal, light, $\mathrm{pH}$, and salt stimuli were mostly investigated in the past few years although

211 other triggers, such as magnetic ${ }^{55}$ or electric fields, ${ }^{56-58}$ can also be used to stimulate the

212 mechanical properties. In the following, we will review relevant examples of smart

213 hydrogels whose mechanical properties can be switched by different signals and discuss

214 their characteristics in detail.

\section{2. Thermo-responsive hydrogels}

216 Among the possible environmental stimuli, the temperature is certainly the most widely

217 used trigger to adjust the mechanical performance of hydrogels. Short response time, easy

218 to operate, and adjustable transition temperatures account for its broad application.

219 According to the toughening mechanisms, temperature-responsive, or more often termed

220 as thermo-responsive hydrogels with stimulated mechanical performance can be classified

221 into three main categories: hydrogels with polymer phase separation, hydrogels with

222 glassy-like transition, and hydrogels with fusible links.

$223 \quad 2.1$ Thermo-mechanical response induced by polymer phase separation.

224 Phase transition in polymers plays a central role not only in the scientific understanding of

225 condensed matter but also in their technological development. ${ }^{49}$ In macromolecular 
226 solutions or for swollen polymer networks, the solubility, and swelling properties,

227 respectively, strongly depend on the polymer/solvent interactions. For a given binary

228 system, polymer/solvent, these interactions vary with temperature and these variations can

229 lead in certain cases to a continuous or discontinuous phase transition around a critical

230 temperature. ${ }^{59}$ Crossing the phase transition temperature $\left(\mathrm{T}_{\mathrm{c}}\right)$, polymer solutions as well as

231 covalent hydrogels experience a tremendous change in viscoelastic properties. Based on

232 the change of their solubility with temperature, thermo-responsive polymers can be

233 classified into two opposite categories: polymers with a Lower Critical Solution

234 Temperature (LCST) or an Upper Critical Solution Temperature (UCST).

235 2.1.1 LCST-type thermo-responsive mechanical hydrogels

236 Although this is not really the phase transition temperature that can be predicted from the

237 classical Flory-Huggins theory for polymer solutions, LCST is the most widely used

238 thermal transition for polymer in aqueous media due to huge number of polymer/water

239 systems that exhibit this behavior. The LCST is the critical temperature below which the

240 components of the mixture are fully miscible for all compositions. Typically, the polymer

241 chains, in the coil-state in water at low temperature, will collapse into a globular state above

242 the transition temperature and aggregate to form a polymer-rich phase in equilibrium with

243 a dilute polymer solution. Among the large amount of LCST polymers, ${ }^{60} \operatorname{poly}(N-$

244 isopropylacrylamide) (PNIPAm) is by far the most studied since the pioneering works on 
245 the phase transition of gels in aqueous media. ${ }^{61}$ Due to its chemical stability, low toxicity,

246 low $\mathrm{pH}$ dependence, ${ }^{62}$ electroneutrality, and its $\operatorname{LCST}\left(\sim 32^{\circ} \mathrm{C}\right)$ close to body temperature

247 and easily adjustable with comonomers, PNIPAm hydrogels have been considered for 248 many applications.

249 As a pioneering work, Takigawa et al. ${ }^{63}$ demonstrated that Young's modulus of homo250 PNIPAm hydrogel was increased by more than one decade upon heating above $\mathrm{T}_{\mathrm{c}}$. During 251 phase separation, the PNIPAm chains self-associate and form a rich-polymer percolating 252 framework which serves as a load-bearing phase to increase gel stiffness, extensibility, and 253 toughness by enhancing energy dissipation (Figure 3a). A linear stress-strain behavior was 254 observed for PNIPAm hydrogel, both below and above the LCST, while later this linearity 255 was observed only at small strains. ${ }^{64}$ Thereafter, many efforts were devoted to the thermo256 responsive toughening of PNIPAm hydrogels. ${ }^{65-67}$ Recently, by incorporating monomers or 257 other organic or inorganic compounds into the PNIPAm network (copolymers, ${ }^{68}$ 258 topological hydrogel ${ }^{69-71}$ or nanocomposites ${ }^{72}$ ) the resulting soft materials have 259 demonstrated thermo-toughening performance comparable to homo-PNIPAm hydrogels. 260 In these cases, the transition temperature of hydrogels can be finely tuned with the 261 incorporation of the other component, no matter chemically copolymerized in the PNIPAm 262 network or physically mixed, ${ }^{73-74}$ to meet the demand of specific applications. In this 263 context, the mechanisms of volume-phase transition in polymer gels have been intensively 264 discussed, both experimentally and theoretically, from the viewpoint of structure, dynamics, 
265 kinetics, and equilibrium thermodynamics. ${ }^{75}$ For more details, readers may refer to the 266 recent review elsewhere. ${ }^{76}$

267 During micro-phase separation, the coil/globule transition undergone at the molecular level 268 by the elastically active PNIPAm chains usually gives rise to a macroscopic shrinkage of 269 the gels, termed as "volume phase transition". ${ }^{50}$ Although this feature can endow the 270 materials with potential applications in specific fields such as sensors and actuators, it 271 hinders many practical perspectives relative to mechanical properties. For example, it is 272 sometimes unnecessary or even undesirable to have a soft material that swells or shrinks 273 during use. In addition, the volume contraction that accompanies the phase separation 274 complicates the interpretation of the exact role of the two-phase morphology on the 275 mechanical properties. It is indeed difficult to separate the intricate contributions of the 276 phase separation and the change in polymer concentration. ${ }^{50}$

277 To solve this puzzle, Guo et al. developed highly swollen networks with different 278 topologies capable of undergoing phase separation with significant modification of their 279 mechanical properties without any change of their macroscopic dimensions (phase 280 transition in isochoric conditions). A first topology was designed by grafting hydrophilic $281 \operatorname{poly}(N, N$-dimethylacrylamide) (PDMA) side chains on a thermo-responsive PNIPAm 282 network (PNIPAm-g-PDMA gel or GPN-D) (Figure 3b). Above $\mathrm{T}_{\mathrm{c}}$, the thermo-responsive 283 PNIPAm backbone phase separates and forms a concentrated load-bearing scaffold 
284 accounting for less than $10 \%$ of the whole volume, while the swelling pressure of the 285 hydrophilic PDMA side chains allows to maintain the high level of hydration of the gel 286 avoiding any volume phase transition. As shown in Figure 3c, the gel effectively retains its 287 original dimensions set by the preparation state on either side of $\mathrm{T}_{\mathrm{c}}$. Unlike conventional 288 covalent bonds, the physical interactions between PNIPAm backbones triggered by the 289 phase separation process have low selectivity and can easily break and reform, ensuring 290 topological adaptation of the stretched network as well as a greater ability to dissipate 291 energy. From small-angle neutron scattering (SANS), it was shown that this gel gave rise 292 to a bi-continuous structure that aligned under loading at high temperature. By comparison 293 with the mechanical properties at low temperature, at high temperature the hydrogels 294 achieved fracture stress and an elastic modulus 10 times higher and extensibility twice as 295 large (Figure 3d). The deformation of the rich-PNIPAm phase also leads to significant 296 dissipative processes and high toughness (fracture energy $\sim 1000 \mathrm{~J}^{-\mathrm{m}^{-2}}$ ). Interestingly, this 297 was also the first time that an unusual crack bifurcation pattern has been reported in the 298 field of hydrogels; such behavior having been observed mainly with natural rubbers (Figure $2993 \mathrm{e})$.

300 During subsequent works, it was shown that the mechanical performances of these thermo301 responsive hydrogels were strongly dependent on the architecture of the networks. ${ }^{77-79}$ In 302 particular, the length and density of hydrophilic grafts are crucial: shorter PDMA chains 303 lead to much less pronounced fracture resistance, while higher graft density results in lower 
304 fracture energy due to the lower fraction of PNIPAm domains (Figure 3f). By comparison,

305 a hydrogel of opposite topology was prepared with the same PNIPAm/PDMA weight ratio

$306(50 / 50)$ but this time by introducing PNIPAM grafts onto the crosslinked PDMA network

307 (GPD-N gel). While exhibiting a similar phase separation process upon heating induced by

308 PNIPAm side chains, this hydrogel showed much lower resistance to fracture than the

309 previous GPN-D gel with no evidence of crack bifurcation. ${ }^{77}$ From SANS experiments, a

310 micellar architecture has been proposed with a swollen PDMA network physically

311 crosslinked via PNIPAm aggregates. With this topology, the thermo-reinforcement

312 increases with the size of PNIPAm sidechains, keeping constant the weight ratio

313 PNIPAm/PDMA. ${ }^{79}$ In fact, this strategy of reinforcement induced by a microphase

314 separation process is somehow similar to that used in the case of nanocomposite gels where

315 macromolecular chains are strongly anchored to the surface of inorganic particles. ${ }^{80}$ The

316 main difference, in the case of GPD-N gels, is that it is the chains of PNIPAm that self-

317 associate to form their own reinforcement domains. In all cases, these organic or inorganic

318 "fillers" act as additional crosslinkers increasing the elastic modulus but also the

319 dissipation phenomena to pull off the chain either from the surface of the inorganic particle

320 or from the polymer microdomain.

321 Compared to previous grafted architectures, other hydrogels such as semi-interpenetrated 322 networks (semi-IPN) have also demonstrated similar thermo-responsive mechanical 323 performance by forming comparable microstructure and giving rise to a similar mode of 
crack propagation. ${ }^{78}$ From a general point of view, the main characteristics of the thermoreinforcement demonstrated with the grafted gels are reproduced with the semi-IPNs and the best properties are obtained with the semi-IPN prepared from a crosslinked PNIPAm network. It is therefore important to form during the phase separation a percolating network

328 (here the crosslinked PNIPAm) as thick as possible which will play the key role of the load-

329 bearing scaffold while keeping in mind that the hydrophilic counterpart remains important

330 to preserve a high level of hydration at high temperature.

331 As the topology of hydrogels has a strong impact on thermo-responsive behavior, the 332 precise construction of network structure is essential for the development of hydrogels with 333 improved mechanical properties. Recently, Ida et al. ${ }^{81-85}$ prepared a series of PNIPAm334 based hydrogels by post-polymerization method, revealing that the type of copolymer 335 network, the feed molar ratio, and the sequence of monomers effectively affected the 336 hydrogel structure, the sharpness of the thermodynamic transition, and the mechanical 337 properties. Well-designed amphiphilic structures with crosslinked PNIPAm domains were 338 formed by post-crosslinking the end-blocks of triblock prepolymers with PNIPAm block 339 either at the middle or at the ends. Compared to the hydrogels with a random sequence of 340 monomers and crosslinkers with the same composition, Ida's hydrogels showed a faster 341 and sharper volume phase transition upon temperature change as well as higher elastic 342 modulus. ${ }^{81-82}$ Thermally responsive crosslinked PNIPAm domains collapse when heated 343 and they act as fillers by dissipating energy and improving hydrogel toughness as 
344 previously described. Interestingly, the amphiphilic hydrogels designed with these thermo-

345 responsive crosslinked domains can be studied under the preparation state while preserving

346 isochoric conditions on either side of the phase transition temperature. Under these

347 conditions, it is possible to specifically analyze the impact of the phase transition on the

348 elastic modulus and the extensibility during heating. ${ }^{81}$

349 While hydrogels derived from PNIPAm have drawn considerable attention to thermo350 responsive toughening performance, some weaknesses remain. One of the major 351 drawbacks of these gels concerns their relatively weak mechanical properties, in particular 352 their low elastic modulus $(\sim$ a few tens of $\mathrm{kPa})$ and fracture stress $(<100 \mathrm{kPa})$ even above 353 the transition temperature. Moreover, while the PNIPAm chain undergoes a relatively sharp 354 coil/globule transition on a small temperature scale, ${ }^{86}$ the corresponding hydrogels undergo 355 a much smoother and gradual mechanical toughening, giving rise to a large hysteresis 356 between heating and cooling processes. This kinetic behavior is closely dependent on the 357 rate of temperature change. It is mainly due to the low collective diffusion coefficient of 358 PNIPAm chains forming the 3D network, as well as to a strong slowing down of their 359 mobility during the formation of very concentrated domains. ${ }^{74}$ This is the case for example 360 with the fracture energy of GPN-D hydrogels which increases from 100 to $1000 \mathrm{~J} . \mathrm{m}^{-2}$ over 361 a wide temperature range from 33 to $60{ }^{\circ} \mathrm{C} .{ }^{87}$ 
a)

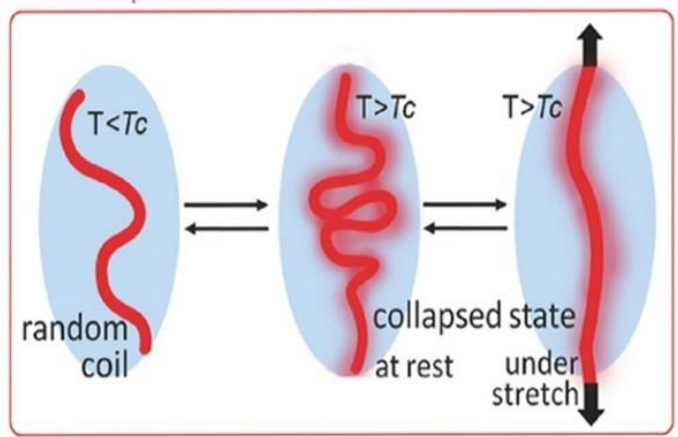

b)

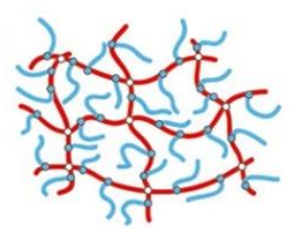

GPN-D
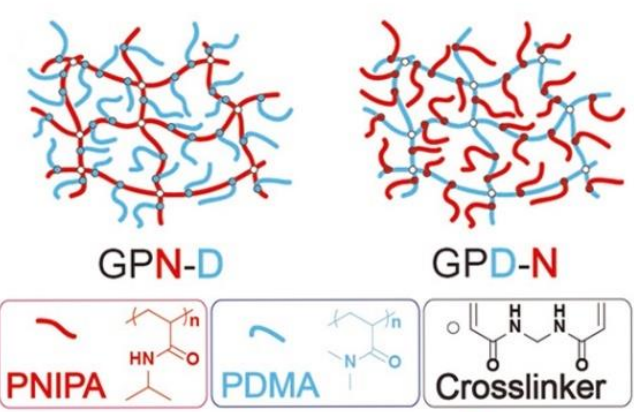

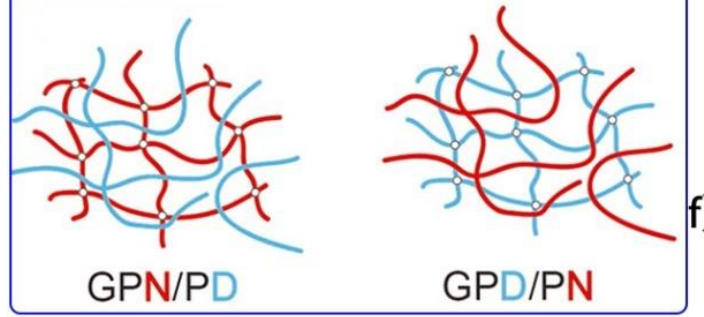

c) GPN-D gel:

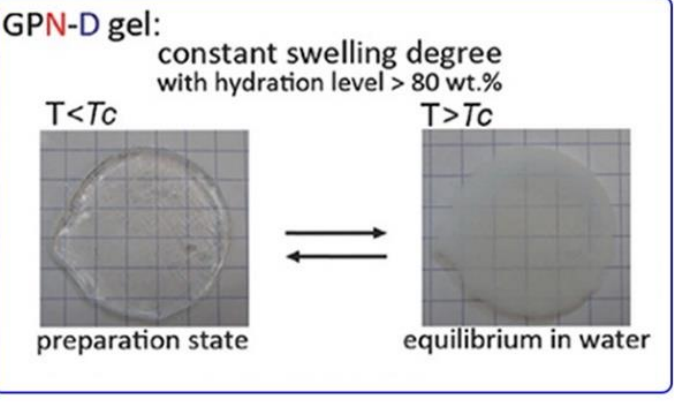

d)

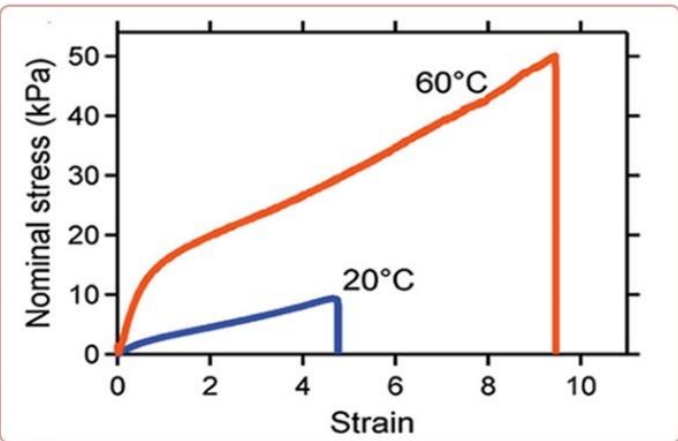

e)
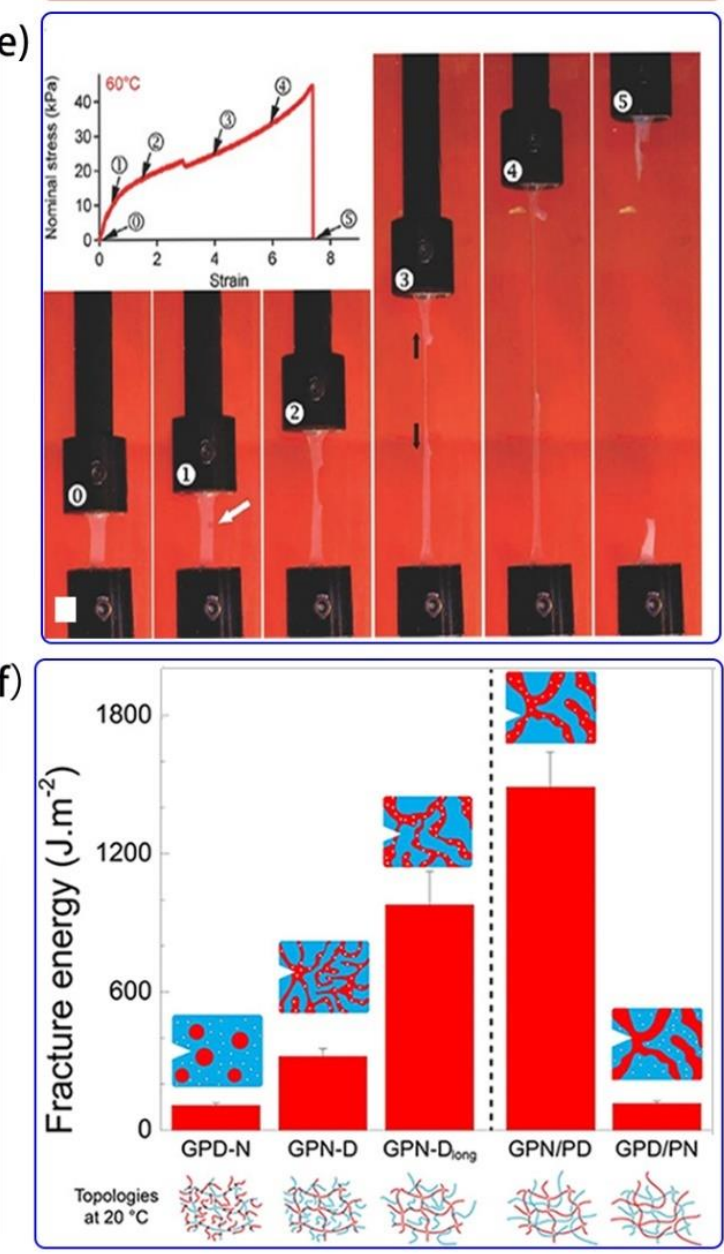

Figure 3. LCST-type thermo-responsive toughening of hydrogels. a) Schematic diagram representing the conformation of PNIPAm at different temperatures. The collapsed PNIPAm chains are defined as the load-bearing phase. Reproduced with permission. ${ }^{87}$ Copyright 2016 WILEY-VCH Verlag GmbH \& Co. KGaA, Weinheim. b) Schematic structure of thermoresponsive graft hydrogels GPN-D, GPD-N, and semi-IPN hydrogels GPN/PD, GPD/PN. Reproduced from ${ }^{78}$ Copyright 2016 American Chemical Society. c) GPN-D gel is able to avoid 
volume phase transition with relatively high hydration level (water content $\cong 83 \mathrm{wt} \%$ ).

370 Reproduced with permission. ${ }^{87}$ Copyright 2016 WILEY-VCH Verlag GmbH \& Co. KGaA,

371 Weinheim. d) Uniaxial tensile stress-strain curves of GPN-D at $20^{\circ} \mathrm{C}$ and $60^{\circ} \mathrm{C}$. e) Photos of GPN-

372 D gels at $60{ }^{\circ} \mathrm{C}$ undergoing fracture test, which illustrate the crack bifurcation process.

373 Reproduced with permission. ${ }^{87}$ Copyright 2016 WILEY-VCH Verlag GmbH \& Co. KGaA,

374 Weinheim. f) Fracture energies obtained with PNIPA/PDMA hydrogels at $60{ }^{\circ} \mathrm{C}$. Reproduced

375 from ${ }^{78}$ Copyright 2016 American Chemical Society.

Besides the thermo-responsive PNIPAm-based hydrogels which are by far the most studied, other LCST-type hydrogels have also demonstrated similar toughening behavior in response to the thermal trigger. Shibayama et al. have recently reported the synthesis and characterization of poly(oligo-ethylene glycol methyl ether methacrylate)-based gels prepared with two monomers having different ethylene oxide chain lengths $(\mathrm{n}=2$ and $\mathrm{n}=$ 4-5). ${ }^{88}$ By changing the comonomer ratio, they were able to change the LCST of the polymer network as well as the association process above the transition temperature providing a large spectrum of elastic to viscoelastic transitions with temperature. Although this study was carried out under isochoric conditions, giving rise to specific information on the biphasic morphology, the analysis of the mechanical properties was limited to small deformation. As reported with PNIPAm-based hydrogels, ${ }^{77}$ the comparable shear modulus obtained at small strain and high temperatures could hide large differences in fracture energies (Figure 3f). Therefore, it seems important to systematically extend the analysis to 390 large deformations to deepen the mechanical characteristics of gels and in particular the 
impact of dissipative processes on fracture phenomena. Indeed, while the previous reported

392 GPN-D hydrogel exhibits fracture energy 10 times higher than that of its GPD-N

393 counterpart of the same composition at $60{ }^{\circ} \mathrm{C}$, they exhibit similar elastic behavior when

394 studied at small deformation. ${ }^{78}$

395 2.1.2 Thermo-responsive mechanical properties of UCST-type hydrogels

396 In contrast to LCST type hydrogels, thermo-responsive polymer networks with an upper 397 critical solution temperature (UCST) can dissolve in solvents above the UCST and start to 398 phase separate below the UCST. In theory, many polymers show a UCST in a given solvent 399 due to complex temperature dependences of the interaction parameter. ${ }^{89}$ The key is whether 400 the thermo-triggered phase transition takes place under gentle conditions as expected for 401 hydrogels (typically between $0-100^{\circ} \mathrm{C}$ ). UCST-type polymers, which are based on specific 402 interactions (hydrogen bonding and/or electrostatic interactions), can be mechanically 403 reinforced by cooling below $\mathrm{T}_{\mathrm{c}}$ and weakened by heating above the transition temperature. 404 Compared to LCST-type hydrogels, UCST ones remain much less explored mainly due to 405 the few available polymer candidates. ${ }^{89-90}$

406 As a representative example of UCST-type polymers, poly( $N$-acryloylglycinamide $)$ 407 (PNAGA) was discovered over half a century ago and widely used in supramolecular 408 chemistry. ${ }^{91}$ Upon heating, highly concentrated PNAGA solutions demonstrate interesting 409 mechanical performance with good stability and thermo-thinning properties. ${ }^{92}$ Interestingly, 
410 by anchoring LCST-type PNIPAm side-chains to a UCST network of PNAGA, Guo et al. ${ }^{93}$

411 have developed new thermo-responsive hydrogels with double temperature response. By

412 adjusting the composition between PNAGA and PNIPAm, it is possible to work in

413 isochoric conditions while exploring the two independent phase transitions (UCST and

414 LCST). As a result, the temperature range can be divided into three different domains: 1)

415 low temperatures ( $<$ UCST and LCST), 2) intermediate temperatures (UCST $<\mathrm{T}<$

416 LCST), and 3) high temperatures ( $>$ UCST and LCST). In the case of this dual thermo-

417 responsive network, the mechanical modifications triggered by opposite phase transitions

418 are dissimilar. At high temperature (T > UCST and LCST), the PNIPAm side-chains

419 collapse and self-associate into concentrated domains through hydrophobic interactions

420 coupled with intra- and inter-molecular hydrogen bonds. These strong interactions give rise

421 to the enhancement of both rigidity and extensibility as already described with hydrophilic

422 PDMA network grafted with PNIPAm side-chains. By comparison, the phase separation of

423 PNAGA at low temperature $(\mathrm{T}<\mathrm{UCST}$ and LCST) does not involve hydrophobic

424 interactions and the intra- and inter-chain associations related to the formation of hydrogen

425 bonds are weaker due to the stronger competition from water molecules for binding sites.

426 The phase transition of the PNAGA network leads to an increase of the Young modulus,

427 similar to that observed at high temperature with the phase transition of the PNIPAm chains,

428 but to a strain at break 10 times lower (Figure 4a). This clearly underlines the importance

429 of exploring the mechanical properties of gels at both small and large deformations. Indeed, 
431 including zwitterionic ${ }^{94}$ or neutral ${ }^{95}$ systems. In addition to changes in mechanical 432 properties, PNAGA-based dual thermally responsive hydrogels ${ }^{96}$ also exhibit the obvious 433 transparency variation upon temperature disturbing, which makes it possible as an 434 electronic skin to qualitatively evaluate the ambient temperature (Figure 4b).

a)
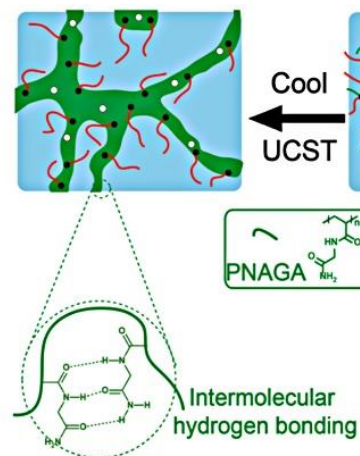

b)

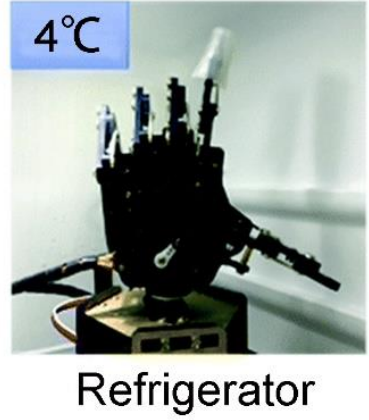

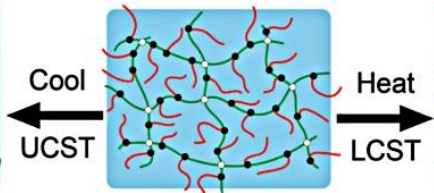
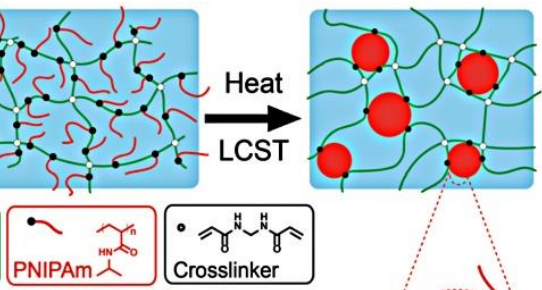
hydrogen bonding

Hydrophobic interactions
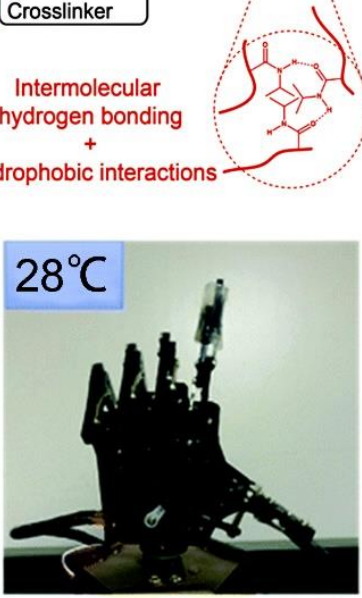

Room temperature
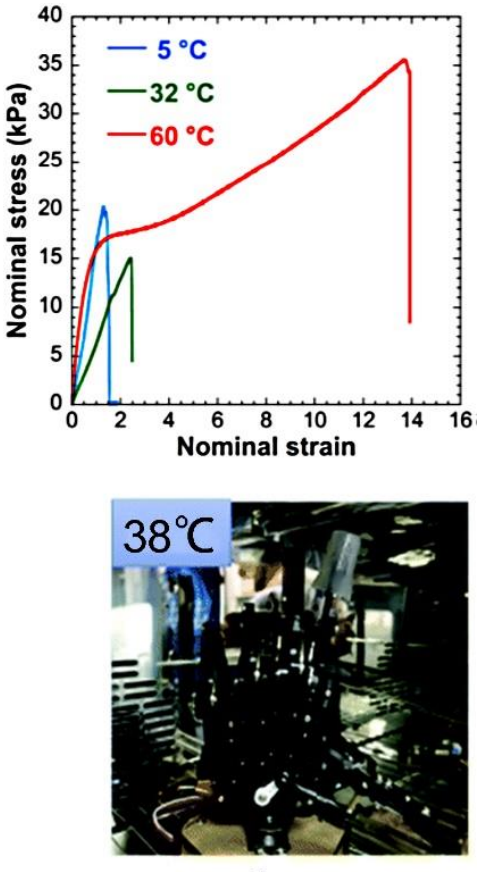

Oven

Figure 4. UCST-type thermo-responsive toughening of hydrogels. a) Schematic representation of thermo-responsive self-assembly and mechanical properties of PAN (NAGA monomer and PNIPAm) hydrogels upon temperature change. Figure colored for better illustration. Original in black and white. Reproduced with permission. ${ }^{93}$ Copyright 2017 WILEY-VCH Verlag GmbH \& Co. KGaA, Weinheim. b) Hydrogel as the robot's electronic skin to test different environment temperatures (refrigerator, room temperature, and oven). Reproduced with permission.96 Copyright 2021 The Royal Society of Chemistry. 
444 In addition to these synthetic systems, similar strategies have been developed on the basis

445 of single or double networks by coupling covalent bonds with physical interactions

446 involving bio-based molecules or polymers capable of self-associating by cooling. This is

447 the case for example with low molecular weight gelators combining guanosine, borax, and

448 potassium hydroxide which can form a supramolecular network within a covalent hydrogel

449 of PDMA, ${ }^{97}$ with crosslinked polyacrylamide networks interpenetrated with agarose chains

450 which are known to reversibly form physical gels upon cooling ${ }^{98-99}$ or with covalently

451 cross-linked gelatin capable of forming additional physical junctions (triple helices) by

452 cooling. ${ }^{100}$ All these studies have demonstrated that the formation of second physical

453 network results in a dramatic increase in strength and toughness at room temperature;

454 physical interactions making an essential contribution to the dissipation process. In these

455 cases, heating/cooling cycles have been mainly applied to promote mechanical recovery

456 after large deformations and self-healing properties rather than studying the variation in

457 mechanical properties throughout the thermal transition.

$458 \quad 2.2$ Thermo-responsive mechanical properties of hydrogels with glassy-like transitions

459 While thermo-softening hydrogels involving UCST polymers remain relatively rare, 460 thermo-softening materials are ubiquitous in nature. In the field of the polymer industry, 461 for instance, thermo-softening plastics, more usually termed thermoplastics, are widely 
462 used for manufacturing a wide variety of items as they are easily pliable or moldable above 463 their softening temperature and solidifies upon cooling. At the molecular level, the polymer 464 segments initially in the frozen state acquire mobility when the temperature is raised above 465 the glass transition temperature $\left(\mathrm{T}_{\mathrm{g}}\right)$, switching the polymer materials from the rigid glassy 466 state to the soft rubbery state. ${ }^{101}$ Without a doubt, this feature is not exclusive to non-solvent 467 materials: a global group of researchers has attempted to apply this strategy in the 468 preparation of thermo-softening hydrogels.

469 In recent years, Wu's group has designed a series of robust hydrogels following this 470 strategy. As a typical example, ultra-stiff and tough hydrogels have been prepared by 471 copolymerizing methacrylic acid (MAAc) and methacrylamide (MAAm) in water. ${ }^{102}$ 472 Indeed, the strong interactions between acid and amide groups allow the formation of a 473 dense and robust hydrogen bond network which gives the material temperature-dependent 474 mechanical properties. Below the transition temperature $\left(\mathrm{T}_{\mathrm{c}}\right)$, hydrogels display 475 considerable stiffness due to the glassy behavior of the polymer phase, with a tensile stress 476 of 4-11 MPa and strain of $400-600 \%$. The sharp decrease in tensile stress detected above $477 \mathrm{~T}_{\mathrm{c}}$ gives these materials mechanical shape memory properties (Figure 5a). Later, the 478 structure-properties relationships and the kinetics of the structure formation have been 479 further investigated to reveal the mechanism of thermo-responsive toughening. ${ }^{103}$ 480 Moreover, systematical researches involving dynamic hydrogen bonds have been carried 481 out to illustrate the concept of glass transition hydrogels as well..$^{93,94}$ 
482 More generally, hydrogels with similar thermo-softening performance have been reported 483 in other systems with various noncovalent interactions, such as hydrogen bonds, ${ }^{104}$ 484 hydrophobic interactions, ${ }^{105-106}$ and ionic bonds. ${ }^{107-109}$ For example, Wang ${ }^{104}$ has reported thermo-responsive poly(vinylpyrrolidone-co-acryloxy acetophenone) glassy hydrogels with high tensile strength $(8.4 \mathrm{MPa})$ and Young's modulus $(94 \mathrm{MPa})$ at room temperature.

487 When heated to $90{ }^{\circ} \mathrm{C}$, the elastic modulus of gels decreased over more than 3 decades 488 making this rigid hydrogel a suitable candidate as a shape memory material for surgical 489 fixation devices to wrap around and support various shapes of limbs. In another study, 490 hydrogen bonds, dipole-dipole, and hydrophobic interactions were coupled by 491 copolymerizing acrylonitrile with $\mathrm{N}$-acryloyl-2-glycine. These tough hydrogels clearly 492 demonstrate thermo-softening performance when heated from $10{ }^{\circ} \mathrm{C}$ (Young's modulus 430 $493 \mathrm{MPa}$ ) to $37^{\circ} \mathrm{C}$ (Young's modulus $16 \mathrm{MPa}$ ). Due to the presence of carboxylic groups in 494 the copolymer network, these hydrogels exhibit a shape memory responsive behavior, 495 controllable by both temperature and $\mathrm{pH}$, preparing them as attractive candidates for 496 permanent embolic agents. ${ }^{110}$

497 While most of this work of hydrogels associates the temperature dependence of mechanical 498 properties with the existence of a glass transition, as judged by the softening performance, 499 it is still unclear whether this transition is really of the same nature as that reported with 500 thermoplastics. For instance, the relaxation times under $\mathrm{T}_{\mathrm{g}}$ in conventional thermoplastics 501 are essentially much longer than the typical duration of an experiment or a numerical 
502 simulation, ${ }^{111}$ while for some of these so-called "glassy hydrogels" the relaxation time is

503 fairly short (e.g. $\sim 1 \mathrm{~s}^{102}$ ). Theoretically, hydrogels with glass transition behavior should

504 generally be designed with high $\mathrm{T}_{\mathrm{g}}$ hydrophobic polymers. However, scientists can

505 effectively prepare tough hydrogels with rather low glass transition polymer networks,

506 which also demonstrate a glassy-like transition. As a typical example, Liang et al. ${ }^{112}$

507 ingeniously designed a body temperature-responsive hydrogel based on the

508 copolymerization of hydrophobic 2-phenoxyethyl acrylate (PEA, $\mathrm{T}_{\mathrm{g}}$ of polymer $\sim 16{ }^{\circ} \mathrm{C}$ )

509 and hydrophilic acrylamide. Within these hydrogels, the high fraction of hydrophobic

510 monomers is responsible for the low content of water at equilibrium (less than $50 \mathrm{wt} \%$ ), as

511 the aggregation of PEA moieties provides a hydrophobic micro-environment for the

512 adjacent amide groups which further stabilize their intermolecular hydrogen bonds. The

513 scission of dynamic hydrogen bonds between acrylamide units by increasing the

514 temperature is then responsible for the thermo-responsive performance. As shown in Figure

$5155 \mathrm{~b}$, the hydrogel exhibits very high tensile strength at low temperature along with a high

516 yield stress and good extensibility, highlighting high energy dissipation. These mechanical

517 properties are highly temperature-dependent: just after heating by hand for a few seconds,

518 the hydrogel becomes weak without any distinct signature of yielding.

519 Similar to thermoplastics which weakens above $\mathrm{T}_{\mathrm{g}}$, all of the aforementioned hydrogels

520 demonstrate thermo-softening performance, which precludes their use in applications

521 which require high mechanical properties at elevated temperature. Although hydrogels 
522 designed with LCST-type polymers are good candidates for improving mechanical

523 properties by heating, the phase separation kinetics, as well as moderate mechanical

524 performances, requires the system to be further improved. Recently, inspired by the

525 molecular mechanism of thermally stable proteins, Gong et al. ${ }^{30}$ developed a novel thermo-

526 responsive hydrogel that undergoes ultra-rapid, isochoric, and reversible switching from

527 soft hydrogels to rigid plastic-like material with increasing temperature. The materials were

528 prepared from inexpensive and non-toxic poly(acrylic acid) (PAAc) hydrogels containing

529 calcium acetate. The synergistic combination of hydrophobic and ionic interactions leads

530 to phase separation by heating with a subsequent rubbery-to-glassy transition at higher

531 temperature. Crossing the transition temperature, the material's stiffness, strength and

532 toughness increased 1800-, 80-, and 20-folds, respectively, and a thin gel sheet could

533 support a $10 \mathrm{~kg}$ weight (Figure 5c). This work may significantly broaden the scope of

534 thermally stiffened polymer applications, such as for heat absorption or in protective

535 sportswear.

536 
a)
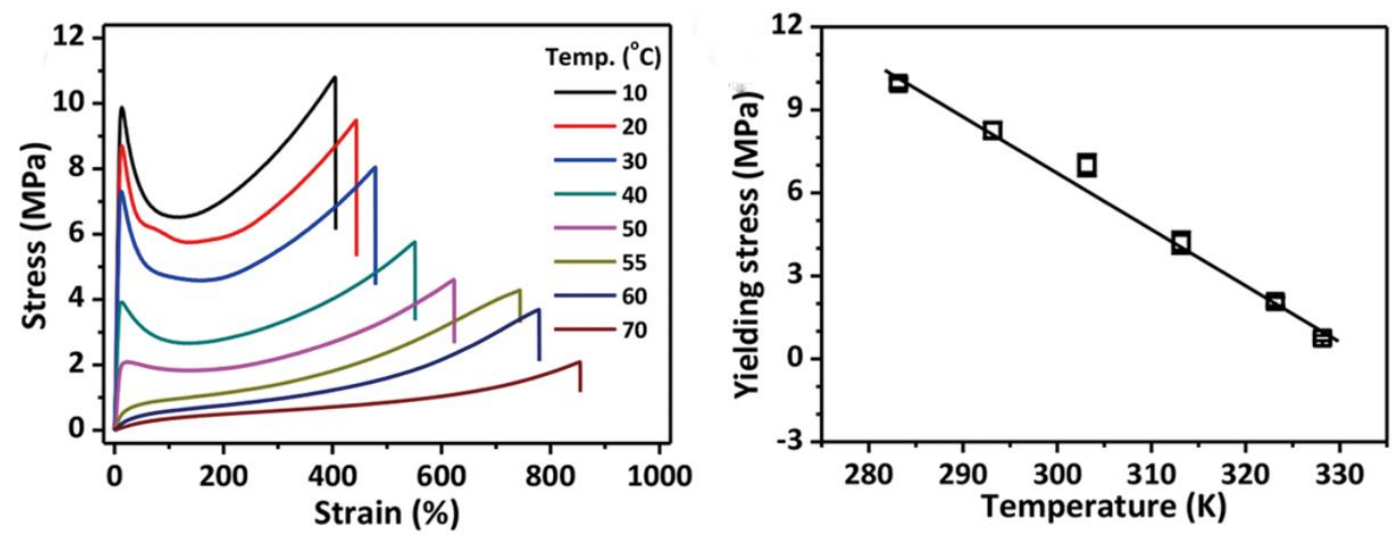

b)
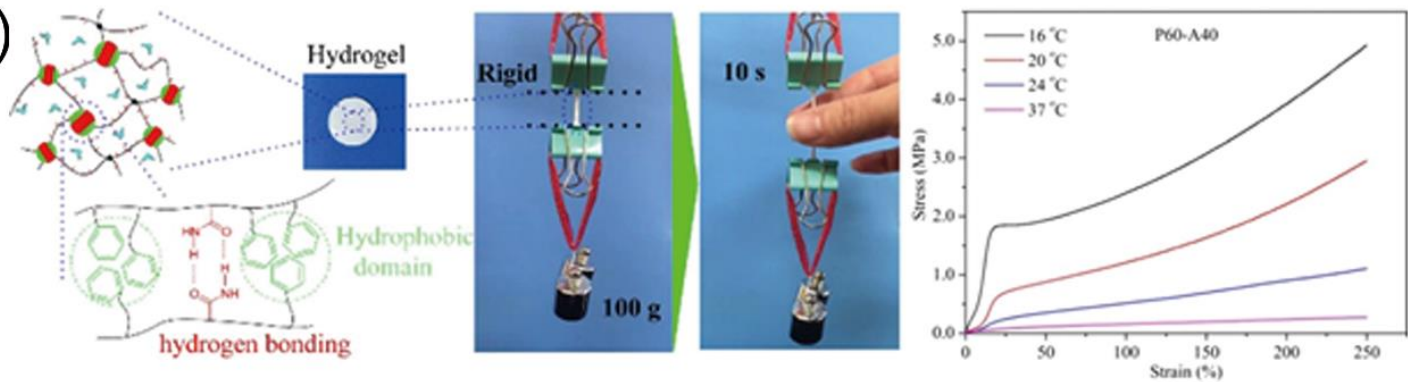

c)
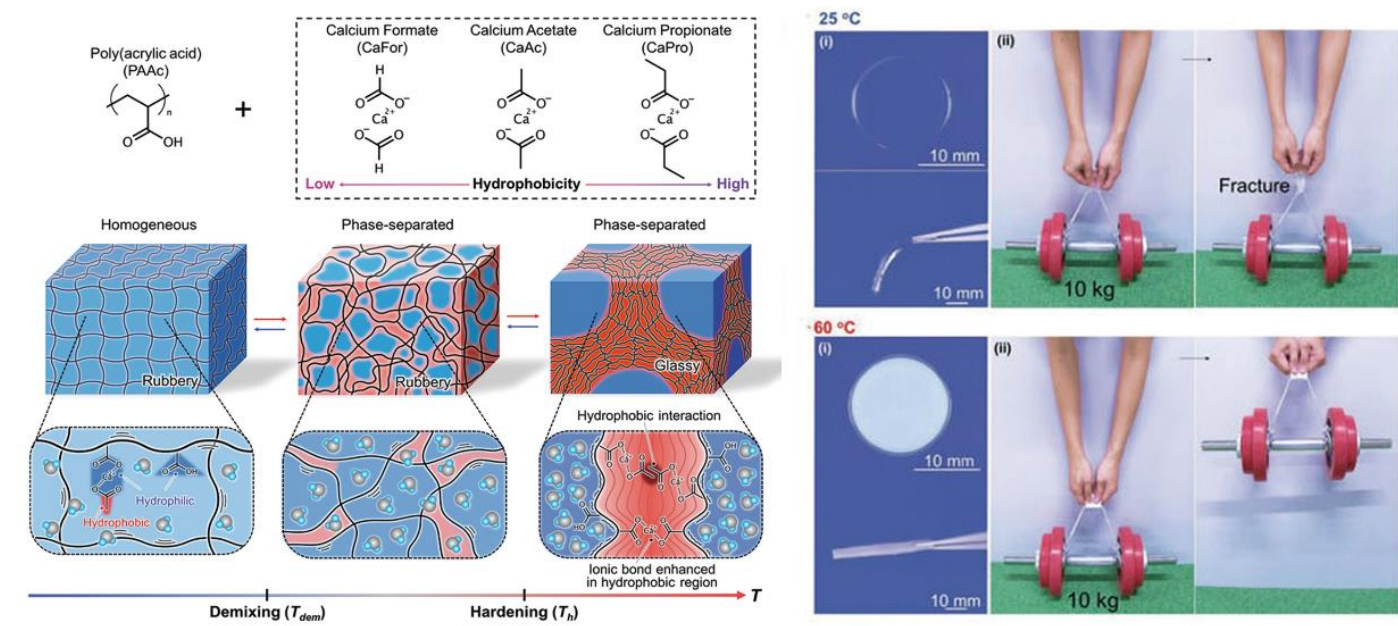

Figure 5. Thermo-responsive hydrogels based on the glassy-like transition. a) Tensile stress-strain curves and yielding stress of poly(methacrylamide-co-methacrylic acid) (P(MAAm-co-MAAc))

540 gel at different temperatures. Reproduced from ${ }^{102}$ Copyright 2019 American Chemical Society. b)

541 Photo illustration of body temperature-dependent mechanical strength and uniaxial tensile

542 behavior of a poly(2-phenoxyethyl methacrylate-co-acrylamide) (P(PEA-co-AAm)) hydrogel with $54360 \mathrm{~mol} \%$ of PEA and $40 \mathrm{~mol} \%$ of AAm (P60-A40). The sheet was rigid at a surrounding 
544 temperature of $10{ }^{\circ} \mathrm{C}$ and could bear a $100 \mathrm{~g}$ weight without any obvious deformation. However, 545 upon heating by two fingers, the hydrogel became soft and was gradually stretched by the loaded 546 weight. Reproduced from ${ }^{112}$ Copyright 2019 American Chemical Society. c) Mechanism of 547 thermo-induced instant switching from soft hydrogel to hard plastic-like materials by 548 polymerizing PAAc hydrogel in presence of calcium ions. Pictures show a load of materials at low 549 and high temperatures, where the gels become significantly toughened up with increasing 550 temperature. Reproduced with permission. ${ }^{30}$ Copyright 2020 Wiley-VCH Verlag GmbH \& Co. 551 KGaA.

554 As we know, crystalline materials are significantly softened by heating above their melting 555 point. This first order thermodynamic transition has also been used to develop thermo556 responsive hydrogels by introducing fusible links in the macromolecular architecture. In this context, several strategies have been considered: either from semi-crystalline networks 558 or by adding crystalline additives. A typical example of such hydrogels was prepared by copolymerization of crystallizable 560 n-stearyl acrylate (SA) segments into a hydrophilic poly(acrylic acid) network. The 561 mechanical properties of these materials exhibit a sharp temperature dependence when the 562 temperature is raised above $50{ }^{\circ} \mathrm{C}$ with Young's modulus of hydrogels decreasing abruptly 563 by about two orders of magnitude from $10^{7}$ to $10^{5} \mathrm{~Pa}^{113}$ Based on Small-Angle X-ray 564 Diffraction and Differential Scanning Calorimetry experiments, it was shown that the long 
565 stearyl side-chains, which self-organize at room temperature into crystalline lamellae 566 aligned perpendicular to the main chain, undergo an order-disorder transition above $50{ }^{\circ} \mathrm{C}$.

567 The melting of these lamellae is then the driving force of the macroscopic transition which

568 allows going from hard plastic to a soft and flexible material. ${ }^{114}$ Similar thermo-responsive 569 gels were prepared by Okay et al. through copolymerizing acrylic acid with 20-50 mol \% 570 of crystallizable n-octadecylacrylate. ${ }^{115}$ In the network, hydrophilic segments provide 571 flexibility to the network while hydrophobic units pack to form crystalline domains that 572 render the material mechanically robust. These melt-processable hydrogels display very 573 high compressive strength (90 MPa) and Young's modulus (26 GPa) as well as shape 574 memory behavior and self-healing properties as expected for such associating systems. In 575 further work, it revealed that the mechanical performance of these semi-crystalline thermo576 responsive hydrogels is related to synthesis parameters, including the type ${ }^{116-117}$ and the 577 amount of the monomers, ${ }^{118}$ the methods of polymerization, ${ }^{119}$ the water content of the 578 hydrogels ${ }^{120}$, and the amount of surfactant. ${ }^{115,121}$ 
a)

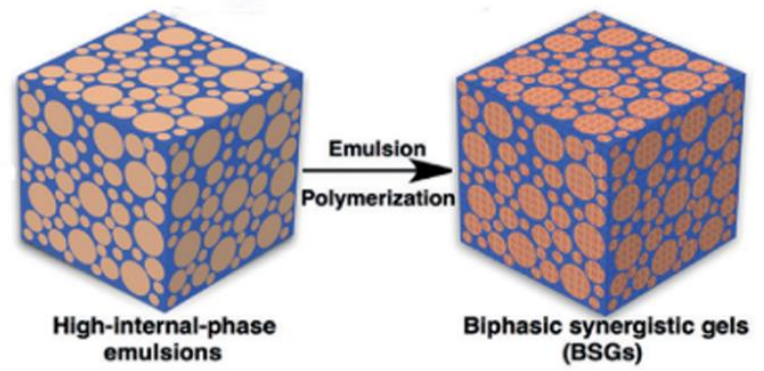
emulsions

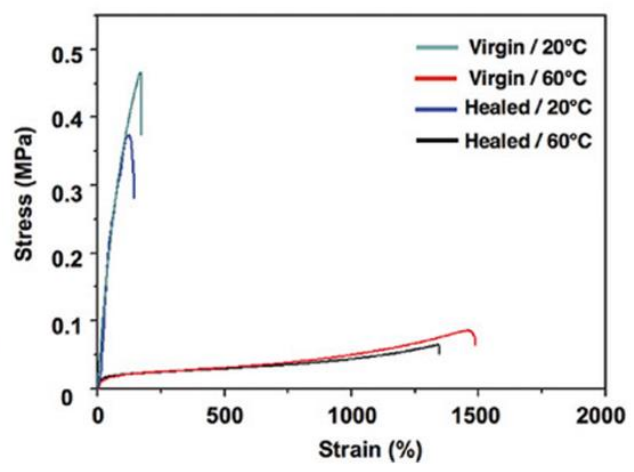

b)
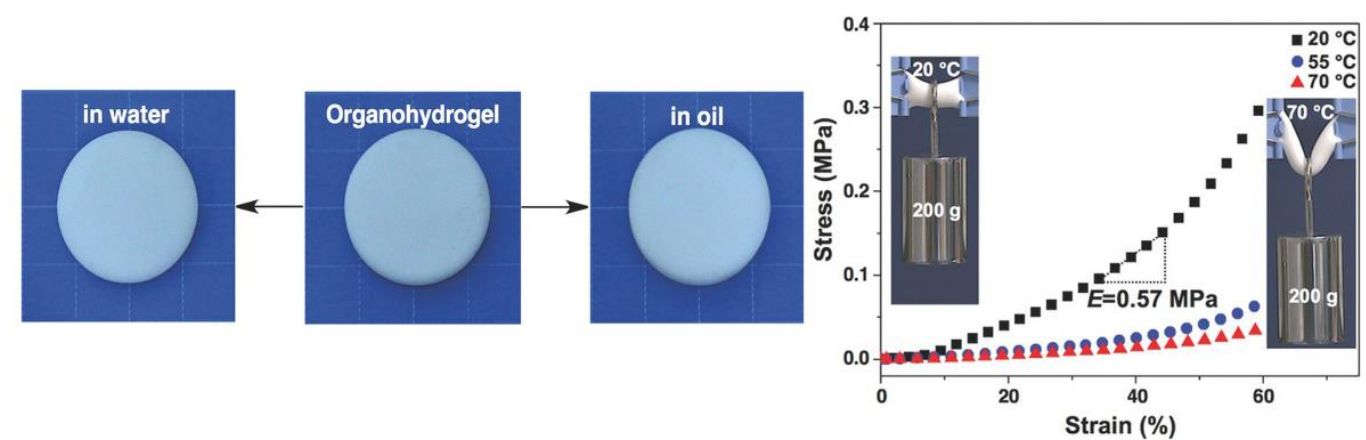

Figure 6. Thermo-responsive hydrogels based with fusible links. a) Scheme and tensile stressstrain curves of virgin and healed biphasic synergistic gels (BSGs) at $20^{\circ} \mathrm{C}$ and $60{ }^{\circ} \mathrm{C}$. Reproduced with permission. ${ }^{122}$ Copyright 2017 Wiley-VCH Verlag GmbH \&Co. KGaA, Weinheim. b) Photos of organohydrogels that exhibit nonswelling behavior in water or oil, and tensile stress-strain curves of organohydrogel at $20^{\circ} \mathrm{C}, 55^{\circ} \mathrm{C}$, and $70{ }^{\circ} \mathrm{C}$. Reproduced with permission. ${ }^{123} \mathrm{Copyright}$ 2017 WILEY-VCH Verlag GmbH \& Co. KGaA, Weinheim.

However, these strategies for preparing thermo-switchable mechanical hydrogels depend on relatively sophisticated molecular design and chemical modification. Although the properties of these hydrogels are rather promising, their method of preparation is far from optimal because it requires several steps with the synthesis in organic solvents, or in water using a large amount of surfactant, followed by a purification step in pure water to obtain 
592 the hydrogels. To overcome this problem, Zhao and coworkers ${ }^{122}$ developed a one-pot 593 strategy to synthesize biphasic synergistic gels, called organohydrogels, by in situ 594 polymerization of oil-in-water high internal-phase emulsions; the oleophilic stearyl 595 methacrylate acting as the oil phase (Figure 6a). The semi-crystalline behavior of closely 596 packed micro-inclusions within the elastic hydrogel matrix not only greatly improves the 597 mechanical properties of hydrogels, but also endows these materials with switchable 598 mechanical behavior. As a result, these gels exhibit a broad spectrum of mechanical 599 performance under tensile tests ranging from high modulus and low strain at break at $20{ }^{\circ} \mathrm{C}$ 600 to low modulus and high strain at $60^{\circ} \mathrm{C}$. In further work, Liu et al. ${ }^{123}$ applied this strategy 601 using lauryl methacrylate as the oily phase, to prepare organohydrogels combining both 602 high strain and tough shape memory properties. These biphasic materials can maintain 603 isochoric conditions, both in water and in oil, and withstanding high load at different 604 temperatures (Figure 6b). ${ }^{123}$ During shape memory experiments, the material was shown 605 to exhibit a high deformation capacity with fully recoverable deformation, both under 606 stretch (up to $2600 \%$ ) and under compression (up to $85 \%$ ) by applying a load almost 20 607 times greater than the weight of the material. In subsequent studies, the introduction of 608 supramolecular interactions (e.g., acrylic acid/ $\mathrm{Fe}^{3+}$ ) in the hydrophilic phase showed that 609 it was possible to prepare organohydrogels responding to two orthogonal stimuli: the 610 temperature for the crystalline oily phase and addition of a competitor for the 611 supramolecular interactions. ${ }^{124}$ 


\section{3. Photo-responsive hydrogels}

614 Like temperature, light is another noninvasive trigger that allows remote, easy, and rapid 615 manipulation of materials without the need of additional reagents, and therefore with

616 limited byproducts. ${ }^{125}$ Typically, the photo-responsiveness of hydrogels can be induced

617 either by the 3D polymer network itself or by inorganic additives incorporated therein.

618 While in the first case the photo-response generally leads to a variation in the degree of

619 crosslinking by photoreaction, the use of additives more generally involves an overall

620 photothermal response without variation in the degree of crosslinking.

\section{$621 \quad 3.1$ Light-responsive polymer networks}

622 Typical photoreactions of polymers include cleavage, ${ }^{40}$ addition, ${ }^{41}$ exchange, ${ }^{42}$ and

623 isomerization. ${ }^{44-45} O$-nitrobenzyl ester is a typical example of cleavage-type functional

624 groups that are currently used for the design of photo-responsive hydrogels. ${ }^{125}$ For instance,

625 the photo-labile bond of the 2-nitrobenzyl functional group of the hydrophobic monomer

626 (2-nitrobenzyloxycarbonylaminoethyl methacrylate (NBOC)), can be irreversibly split

627 under UV irradiation with the formation of hydrophilic 2-aminoethyl methacrylate (AMA)

628 and the release of 2-nitrobenzaldehyde and $\mathrm{CO}_{2} \cdot{ }^{126}$ Starting from a linear copolymer of

629 hydrophilic 2-ureidoethyl methacrylate (UM) and NBOC, poly(UM-co-NBOC), which 
630 initially self-assembles through hydrophobic interactions between NBOC units, UV

631 irradiation will cause a succession of chemical and physical events as follows 1) the

632 transformation of hydrophobic NBOC groups into hydrophilic AMA units, 2) the

633 disassembly of hydrophobic crosslinks and 3) the chemical reaction between amino groups

634 of AMA and ester bonds of UM thus forming new chemical crosslinks. ${ }^{126}$ As shown in

635 Figure 7a, poly(UM-co-NBOC) hydrogels after UV irradiation exhibit a higher Young's

636 modulus but lower failure strain and stress compared with those without UV irradiation.

637 Due to the limited penetration thickness of UV, the hydrogel demonstrates a sandwiched

638 structure with two rigid outer layers and a soft inner layer after UV irradiation. Under

639 deformation, the outer layers fractured preferentially (like the first highly crosslinked

640 network of a double network gel), while the inner layer maintained the whole integrity of

641 hydrogels, playing a role resembling the second loosely crosslinked network of DN gels.

642 By adjusting UV curing time and/or UV penetration length, the precise control of the

643 sandwich structure can be finely achieved. The thinner the soft middle layer, the weaker

644 the ability to bear the stretching and maintain global integrity.

645 Another elegant approach is to prepare hydrogels with photo-tunable crosslinking degrees

646 via photo-addition reactions. Coumarin (COU), one of the well-known light-sensitive

647 molecules, undergoes a [2+2] photo dimerization under irradiation at $365 \mathrm{~nm}$ while the

648 resulting adduct can be decoupled by irradiation at $254 \mathrm{~nm} .{ }^{127}$ Sun et al. ${ }^{41}$ have first

649 prepared linear polyacrylamides bearing pendant COU groups. In the presence of $\gamma$ - 
650 cyclodextrin $(\gamma \mathrm{CD})$, which can host two molecules of $\mathrm{COU}$, the initial polymer solution 651 was transformed into a transient network with the formation of dynamic COU- $\gamma \mathrm{CD}-\mathrm{COU}$ 652 interchain bonds. After irradiation at $365 \mathrm{~nm}$, the dimerization of the $2 \mathrm{COU}$ molecules 653 hosted in the same $\gamma \mathrm{CD}$ cavity transforms the physical bond into a covalent bond which 654 results in stiffening of the network with a doubling of the storage modulus. In contrast, 655 when irradiated at $254 \mathrm{~nm}$ light, the chemical hydrogel turns back to a soft host-guest 656 physical network.

657 In contrast to photo-responsive toughening of hydrogels via the tuning of covalent bonds, 658 the possibility of using dynamic photo-reversible noncovalent interactions has been much 659 less investigated. Very recently, it has been reported that photo-sensitive coordinate bonds 660 can efficiently release the energy stored by the hydrogel. ${ }^{128}$ The authors took 661 poly(acrylamide-co-acrylic acid) (P(AAm-co-AAc)) hydrogel for illustration. After being 662 prestretched and immersed in $\mathrm{Fe}^{3+}$ solution, the deformation of hydrogel is fixed by the 663 coordinate bonds between $\mathrm{Fe}^{3+}$ and carboxyl groups from AAc units which temporarily 664 store potential energy in hydrogels. After UV irradiation, $\mathrm{Fe}^{3+}$ is reduced to $\mathrm{Fe}^{2+}$, resulting 665 in the breakdown of the coordinate bonds and the fast release of the elastic potential energy 666 stored in the hydrogel. ${ }^{128}$ This strategy of storing and releasing elastic energy, based on the 667 destabilization of the coordination bonds by photoreduction, endows hydrogels with an 668 elasticity-plasticity switchability, multi-stable deformability in fully reversible and 669 programmable manners, and anisotropic or isotropic deformations. With the high power 
670 density and programmability via this customizable modular design, these hydrogels

671 demonstrated potential for broad applications in artificial muscles, contractile wound

672 dressing, and high-power actuators. In the same manner, photo-responsive adhesion can be

673 easily achieved by tuning coordination bonds between $\mathrm{Fe}^{3+}$ and carboxyl groups. ${ }^{129}$ Upon

674 irradiation, the dissociation of complexes causes a strong decrease in the adhesion energy

675 from 200 to $10 \mathrm{~J} \mathrm{~m}^{-2}$.

676 However, the above photo-cleavage and addition reactions are generally not fully 677 reversible, which in some cases may limit the practical use of such materials. 678 Comparatively, the use of photo-isomerization reactions makes it possible to prepare 679 materials with satisfactory reversibility of their mechanical properties. Azobenzene, as a 680 photo-responsive molecule, undergoes trans-to-cis and cis-to-trans isomerizations upon 681 exposure to UV and blue light, respectively, resulting in a change of distance between the 682 two phenyl rings (from $9 \AA$ to $5.5 \AA$ ) and dipole moments (from $0.5 \mathrm{D}$ to $3.1 \mathrm{D}$ ) for trans 683 and cis isomers respectively. Hydrogels that incorporate photo-switchable molecules can 684 exhibit reversible variations in stiffness as a function of the irradiation wavelengths. These 685 stimuli-sensitive hydrogels are candidates to direct or probe osteogenic and myogenic 686 differentiation of human mesenchymal stem cells (MSCs). ${ }^{130-131}$ It has been reported that 687 MSCs grown on substrates with varying stiffness express implicit changes, such as 688 development, aging, and fibrosis. Upon blue light $(490 \mathrm{~nm})$, the polyacrylamide hydrogel 689 containing 4,4'-diacrylamidoazobenzene becomes stiffer as the predominant trans isomer, 
690 which is less polar, has a greater ability to self-associate. At the same time, cells exhibit a

691 better spreading with larger spread cell areas in these stiffer hydrogel substrates compared

692 to softer ones (Figure $7 b$ ). ${ }^{132}$ In a similar work, a peptide crosslinker containing azobenzene

693 is introduced into poly(ethylene glycol) to form a light-responsive hydrogel. Upon

694 irradiation with cytocompatible doses of $365 \mathrm{~nm}$ light (UV), isomerization to the

695 azobenzene cis configuration leads to a softening of the hydrogel up to $100-200 \mathrm{~Pa}$ (shear

696 storage modulus). The modulus of the gel can be well tuned upon irradiation with light

697 with different wavelengths (Figure 7c). The planar trans conformation can better stabilize

698 the hydrogen bonds between the peptide cross-linkers and harden the substrate. ${ }^{133}$

a) $\bigcup_{\text {UM }}^{+} \underset{\text { NBOC }}{\stackrel{\text { copolymerization }}{\stackrel{\text { in DMSO }}{\rightarrow}}}$

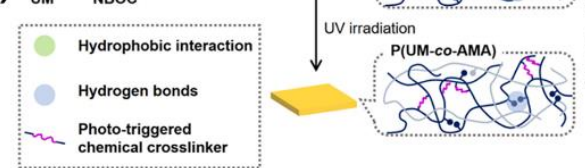
Photo-triggered

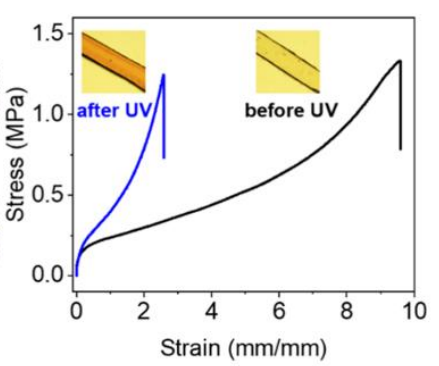

C)

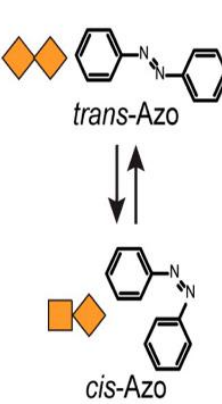

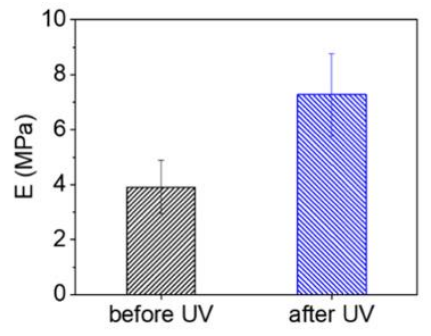

b)
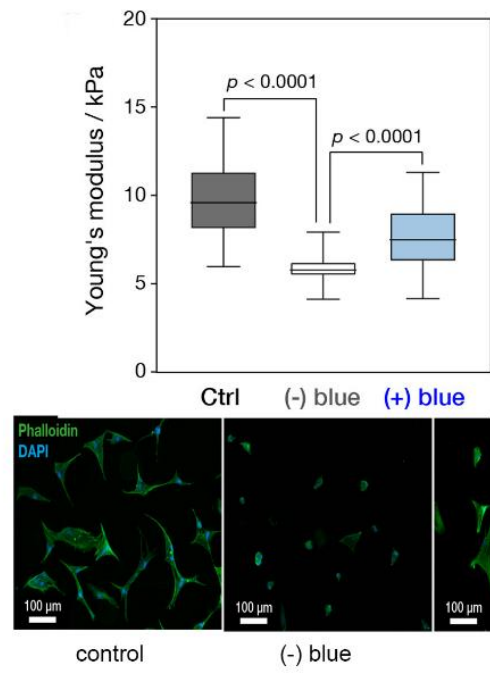

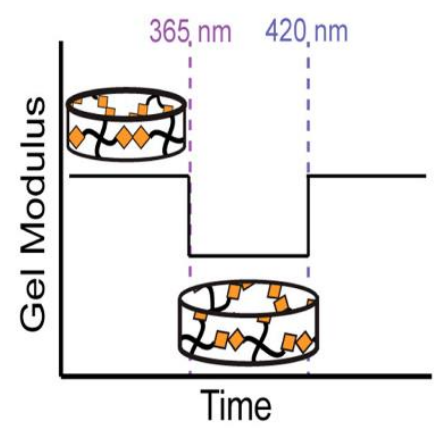


Figure 7. Photo-responsive hydrogels with tunable mechanics. a) Schematic illustration "sandwich" structured hydrogels composed of poly(2-ureidoethyl methacrylate-co-2nitrobenzyloxycarbonylaminoethyl methacrylate) (P(UM-co-NBOC)) (yellow part) and poly(2ureidoethyl methacrylate-co-2-aminoethyl methacrylate) (P(UM-co-AMA)) (gray part) hydrogels. Tensile stress-strain curves and corresponding Young's modulus (E) of the hydrogels before and after UV irradiation are shown on the right. The inset images are cross-sectional micrographs of the sample. Reproduced from ${ }^{126}$. Copyright 2019 American Chemical Society. b) Young's modulus of hydrogel after treatment and fluorescence microscopy images of mesenchymal stem cells (MSCs). Control gels (ctrl) that had not been exposed to UV. Blue gels were softened by exposure to UV (365 $\mathrm{nm})$ irradiation for $30 \mathrm{~min}$; (+) blue gels were exposed to blue (490 $\mathrm{nm})$ light for $1 \mathrm{~h}$, whereas (-) blue gels were unirradiated (left in the dark). Ctrl cells to be well spread, (-) blue cells to be smaller, and (+) blue cells to have recovered spreading. Reproduced from ${ }^{132}$. Copyright 2018 American Chemical Society. c) Isomerization of azobenzene (Azo) group and modulus of gels upon light. Reproduced from ${ }^{133}$. Copyright 2015 American Chemical Society.

\subsection{Light-responsive additives}

Along with the hydrogels described previously, in which the photosensitive molecules belong to the architecture of the polymer network, photo-stimulation properties can also be generated using thermo-responsive polymers and photo-sensitive inorganic fillers. Among the systems investigated, near-infrared (NIR) often serves as a light trigger while gold nanoparticles or nanorods, ${ }^{134-135}$ ferrosoferric oxide nanoparticles $\left(\mathrm{Fe}_{3} \mathrm{O}_{4} \mathrm{NP}_{\mathrm{s}}\right),{ }^{136}$ and graphene oxide $(\mathrm{GO})^{137}$ are generally used as photo-convertor. In response to irradiation, part of the light energy is converted into thermal energy, which is referred to as the 
723 photothermal effect. As an example, Huang and co-authors ${ }^{138}$ developed light- and thermo-

724 responsive hydrogels by dispersing GO into interpenetrating networks formed by

725 physically cross-linked gelatin and chemically cross-linked polyacrylamide. Without light

726 irradiation, relatively high mechanical toughness (strength $>400 \mathrm{kPa}$ and fracture strain $>$

$727500 \%$ ) was achieved by the double-network which dissipates energy through the sacrificial

728 gelatin network and GO particles bridging the two networks through supramolecular

729 interactions. Under the NIR trigger, temperature increases rapidly and causes the

730 dissociation of the thermo-responsive gelatin network which greatly declines the modulus

731 of the gel. With optimal content of GO and enough NIR time, hybrid GO-based hydrogels

732 display thermo-patterning and NIR irradiation erasing performances.

733 In another representative example, $\mathrm{Wu}$ and co-workers have developed stiff and tough

734 shape memory hydrogels responding to NIR light irradiation by incorporating gold

735 nanorods into a glassy gel matrix of poly(1-vinylimidazole-co-methacrylic acid)

736 hydrogels. ${ }^{139}$ Owing to the photothermal properties of gold nanorods, the localized

737 temperature rise induced by the NIR light irradiation, which turns the hydrogel from rigid

738 to soft by crossing the $\mathrm{T}_{\mathrm{g}}{ }^{140}$. After irradiation and cooling to room temperature, the

739 hydrogels show an increase in Young's modulus (E), from 13 to $57 \mathrm{MPa}$, and yielding

740 stress $\left(\sigma_{\mathrm{y}}\right)$, from 0.6 to $2.1 \mathrm{MPa}$, whereas the water content decreases from 71 to $59 \mathrm{wt} \%$

741 (Figure 8a). This behavior is attributed to the collapse of copolymer chains at high

742 temperature (above $\mathrm{T}_{\mathrm{g}}$ ) which leads to the formation of a denser network of hydrogen bonds 
743 during cooling

744 The use of inorganic composite under light irradiation also proves to be an effective way 745 to tune the hydrophilic/hydrophobic balance of the material and consequently adjust the 746 mechanical properties of hydrogels. Recently, a sophisticated formulation of hybrid 747 materials was developed by Park and co-workers via loading in a poly(vinyl 748 alcohol)/chitosan matrix photocatalytic $\mathrm{TiO}_{2} / \mathrm{CD}$ particles (CD for silica-carbon dots) 749 which exhibit a unique light absorption behavior with a wide UV-vis range of absorption 750 wavelengths. In that system, metal oxides $\mathrm{TiO}_{2}$ produces a photoelectric effect under UV 751 activation, while the CDs demonstrate photoelectric properties due to the recombination of 752 photo-excited electron-hole pairs. Under UV or visible irradiation, the photocatalytic 753 nanomaterial changes the surface wettability of the thin-film hydrogel from hydrophobic 754 to hydrophilic due to reactive oxygen $\left(\mathrm{O}^{2-}\right)$ and hydroxide species $(-\mathrm{OH})$ which were 755 formed during the process. This hydrophilicity modification remarkably affects the 756 permeability to water which swells the hydrogel and therefore modifies its mechanical 757 behavior as well as its electronic properties (Figure 8b). 
a)

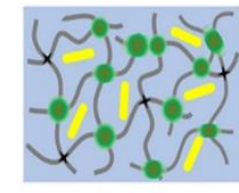

Tough hydrogel

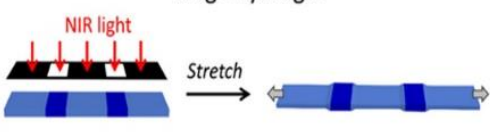

b)

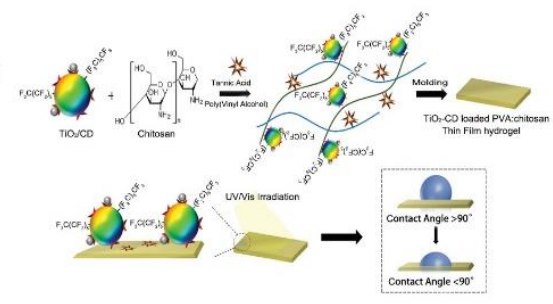

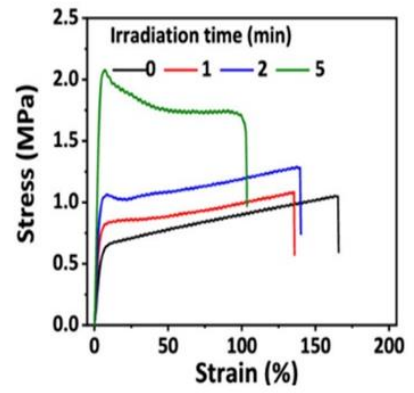
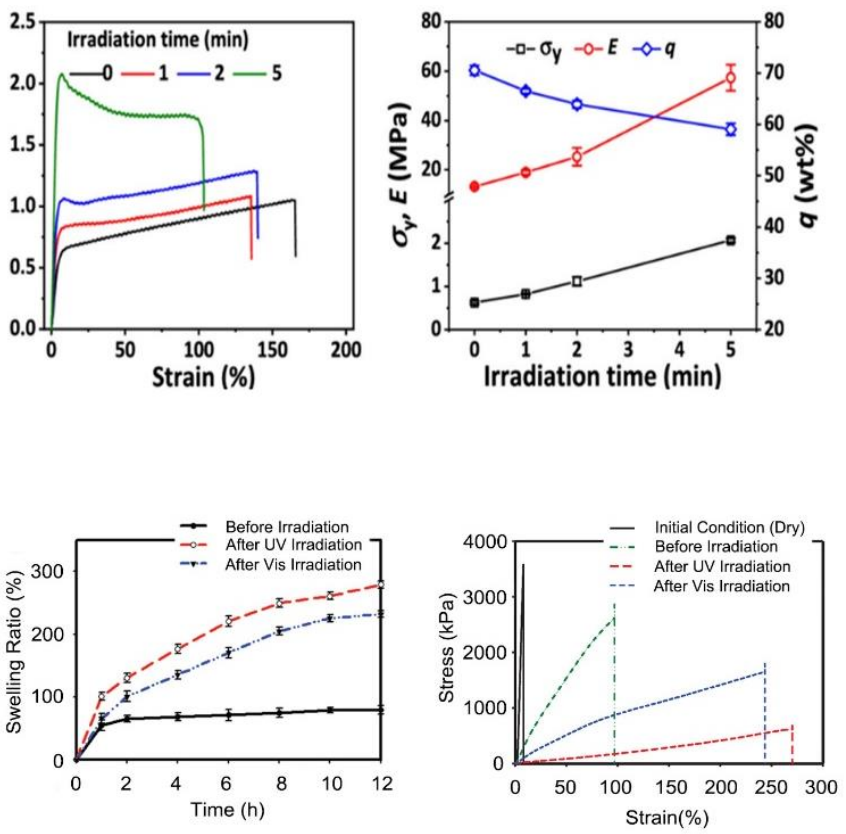

Figure 8. Photo-responsive hydrogels with tunable mechanics induced by embedded additives. a) Schematic and light responsive performance of poly(1-vinylimidazole-co-methacrylic acid) hydrogels integrated with $3 \mathrm{nM}$ of gold nanorods. The tensile stress-strain curves and the corresponding Young's modulus $(E)$ and yielding stress $\left(\sigma_{y}\right)$, as well as the water content $(q)$ determined as a function of irradiation time are shown on the right. Reproduced from ${ }^{139}$. Copyright 2020 American Chemical Society. b) Scheme of the hydrogel thin-film based on titanium oxide $\left(\mathrm{TiO}_{2}\right)$ polydopamine-perfluorosilica carbon dot (CD)-conjugated chitosanpolyvinyl alcohol-loaded tannic acid along with $\mathrm{UV}$ and vis irradiation response affecting the surface hydrophilicity. The force-displacement curve and the swelling ratio of the hydrogel are shown on the right. Reproduced with permission. ${ }^{141}$ Copyright 2019 WILEY-VCH Verlag GmbH \& Co. KGaA, Weinheim. Although sharing various comparable advantages to the temperature stimulus, the light trigger is much less utilized, mainly due to the intrinsic feature of light. On one hand, upon 
773 irradiation with relatively short-wave light such as UV, the short penetration depth is often

774 a critical shortcoming. ${ }^{142}$ In certain cases, the high density also led to the degradation of

775 the network's backbone. ${ }^{143}$ On the other hand, relatively long-wave light such as NIR is

776 often unable to provide enough energy to initiate photoreactions.

\section{4. pH-sensitive hydrogels}

$778 \mathrm{pH}-$ responsive hydrogels are a subset of stimuli-responsive systems capable of responding

779 to $\mathrm{pH}$ environment. Weak polyelectrolyte hydrogels containing partially ionizable acidic

780 units such as acrylic acid or methacrylic acid will become negatively charged with

781 increasing the $\mathrm{pH}$, typically above their $\mathrm{pKa}$, and their counter-ions induce a huge increase

782 of the osmotic pressure. On the other hand, hydrogels containing weak basic groups, like

783 dimethylaminoethyl acrylate or 1-vinylimidazole, become cationic by lowering the $\mathrm{pH} .{ }^{144}$

784 As acidic or alkaline conditions have a significant impact on the strength of physical

785 interactions, such as hydrogen bonds or ligand-metal coordinate bonds, they can be used

786 to tune the macroscopic properties of hydrogels. In addition, the responsive ionization of

787 networks at different $\mathrm{pH}$ also leads to significant volume change with osmotic pressure.

788 This will greatly influence the mechanical properties of the hydrogels but in the following

789 examples the impact of the physical interactions on the elastic properties is much greater

790 than that linked to the variation in volume as given by $G \sim Q^{-b}$ with scaling exponent $b=$

$791 \quad 1 / 3$ in $\theta$ conditions and $b=7 / 12$ in a good solvent. 
793 A hydrogen bond is an electrostatic attraction between polar molecules that occurs when a 794 hydrogen atom is bound to a highly electronegative atom such as nitrogen, oxygen, or 795 fluorine. ${ }^{145}$ These combinations are not stable enough, especially in aqueous media with $796 \mathrm{pH}$ disturbing. For instance, the deprotonation of $-\mathrm{COOH}$ in a basic environment leads to 797 the breaking of the transient network of hydrogen bonds formed by the carboxylic 798 groups. ${ }^{146-147}$

799 Zhang and co-authors reported a $\mathrm{pH}$-sensitive and tough hydrogel composed of poly(1800 vinylimidazole-co-acrylic acid) (P(VI-co-AAc)). The formation of intra- and inter-chain 801 hydrogen bonds between the two monomers endows the gel with significant rigidity and 802 strength at room temperature in a large range of $\mathrm{pH}(3 \leq \mathrm{pH} \leq 10)$ (Figure 9a). The high 803 stability of hydrogen bonds is related to the strength of the proton donor-acceptor pair. ${ }^{148}$ 804 However, the hydrogen bonds can be destructed, either in strongly acidic conditions by 805 ionization of the imidazole function from VI, or under alkaline conditions with ionization 806 of the carboxylic group from AAc. In such extreme conditions, the gels become much 807 weaker. Similar behavior has been reported with other types of gels with strong hydrogen 808 bonds. $^{112,149}$

809 4.2 Metal-ligand coordinate bonds 
810 In a coordinate bond, two electrons from one atom are supplied to a second atom to

811 generate the interaction. Many parameters, such as the electron-donating ability of the

812 ligand and the empty orbital of the central metal, govern the strength of the coordinate

813 bond. Similar to hydrogen bonds, metal-ligand coordinate bonds are typically vulnerable

814 to $\mathrm{pH}$ modifications as it dramatically changes the electron density of ligands or metals.

815 For instance, the ionization of carboxylic groups and the nature of coordination states

816 (mono-, bis-, or tris-ligand-metal complexes) are very sensitive to the $\mathrm{pH}$ environment,

817 which results in a variation of the association constants of carboxyl-Fe ${ }^{3+}$ chelates. ${ }^{37,150}$

818 Under weak acidic conditions $(\mathrm{pH}=4-5)$, the tris-carboxyl- $\mathrm{Fe}^{3+}$ coordinate with the

819 highest stability constant dominates the association behavior and gives rise to the highest

820 stress at break ( $\sigma_{b}$; see Figure $\left.9 b\right)$, Young's modulus and extension work. On the other hand,

821 in more acidic environments $(\mathrm{pH}=1.7-3.5)$, the swelling of the gels increases slightly,

822 while their mechanical properties decrease sharply, indicating the partial destruction of the

823 coordination complexes. Under extreme conditions, gels dissolve in highly acidic medium

$824(\mathrm{pH} \leq 1)$ and disintegrate in alkaline solutions $(\mathrm{pH} \geq 12)$, forming ferric hydroxide

825 precipitates. ${ }^{35}$ Analogous carboxylic coordination complexes have been reported with other

826 metals like $\mathrm{Zr}^{4+},{ }^{151} \mathrm{Cu}^{2+},{ }^{152} \mathrm{Ag}^{+},{ }^{153}$ and $\mathrm{Ca}^{2+152,154}$. For all these hydrogels, the mechanical

827 behavior remains closely related to the nature of the metal and that of the ionizable ligand,

828 as the stability of the coordinate bond strongly depends on the size of the cations. ${ }^{154}$ 
830 In addition to the dynamic binding mechanisms mentioned above, when hydrogels carrying 831 ionizable groups are exposed to acidic or alkaline conditions, they can undergo large 832 variations in volume which strongly impact the mechanical performance. ${ }^{144,146}$ In the case 833 of PAAc hydrogels for example, the low level of swelling at low $\mathrm{pH}$ induced by the 834 formation of hydrogen bonds between carboxyl groups makes the gels rather rigid and 835 stable. Then, by increasing the $\mathrm{pH}$ above the $\mathrm{pKa}$, the transformation of -COOH groups 836 into carboxylate and counterions breaks the hydrogen bonds and generates a strong osmotic 837 pressure leading to highly swollen and very soft gels which may eventually undergo 838 autolysis. Although this is the general trend for weak polyelectrolytes up to moderate 839 swelling, i.e. a Gaussian stretching regime characterized by a decrease of the modulus upon 840 swelling, an abrupt upturn associated with non-Gaussian elasticity is observed for 841 extremely larger swelling ratio $>100)$, i.e. smaller polymer volume fraction $\left(\phi_{2}<0.01\right) .{ }^{155}$ 842 For the same reasons, the protonation at low $\mathrm{pH}$ of the nitrogen atom of dansyl groups, 843 introduced by copolymerization within PAAm hydrogels, increases the swelling ratio by 844 generating high osmotic pressure and therefore make the gels much softer and less 845 stretchable. ${ }^{156}$ Then, when the $\mathrm{pH}$ increases above $\mathrm{pKa}$, the deprotonated and hydrophobic 846 dansyl groups self-associate and act as dynamic crosslinks which improve the toughness 847 of dansyl-based hydrogels (Figure 9c). Together with the $\mathrm{pH}$ and thermo-responsiveness 848 induced by the host-guest complexes, the materials exhibit a triple-shape memory 
849 performance.

850 Although $\mathrm{pH}$ is testified as an effective trigger for tuning the mechanical properties of 851 hydrogels, it is worth mentioning that this environmental trigger is less attractive compared 852 to temperature and light. One of the main reasons is that the response time is longer since 853 it requires the diffusion of solutes and solvent within the network and the volume variation 854 is generally quite large. Indeed, the rate of swelling, which is intrinsically linked to the 855 collective diffusion coefficient of the polymer, is also influenced by the characteristics of 856 the sample itself; in particular its size, shape, and porosity. ${ }^{157}$ Another disadvantage of the $857 \mathrm{pH}$ stimulus comes from implementation issues, which generally require a specific liquid 858 exchange to trigger the mechanical response. 

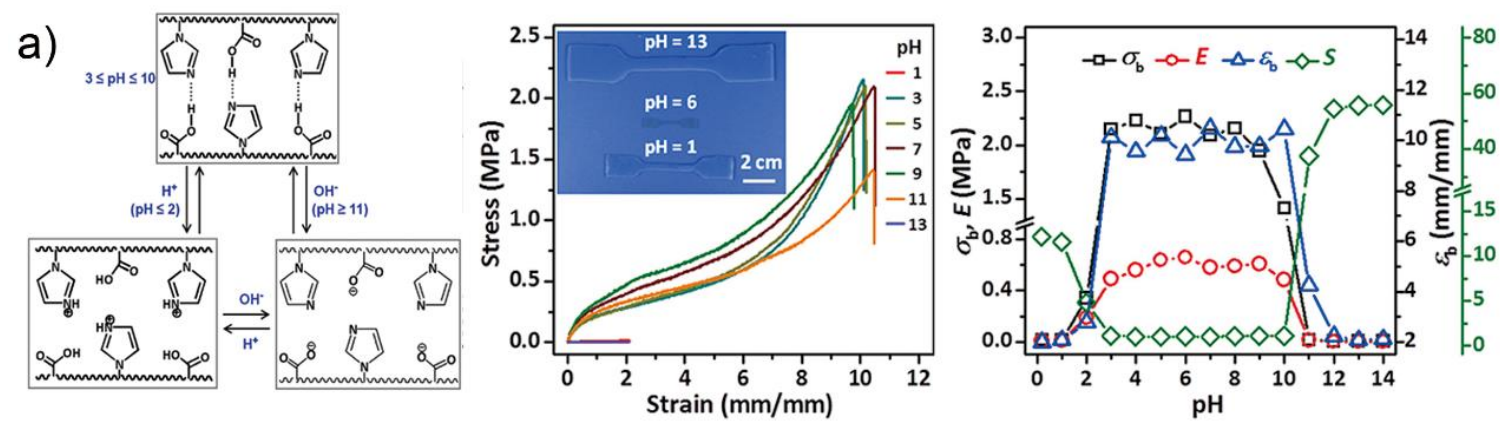

b)
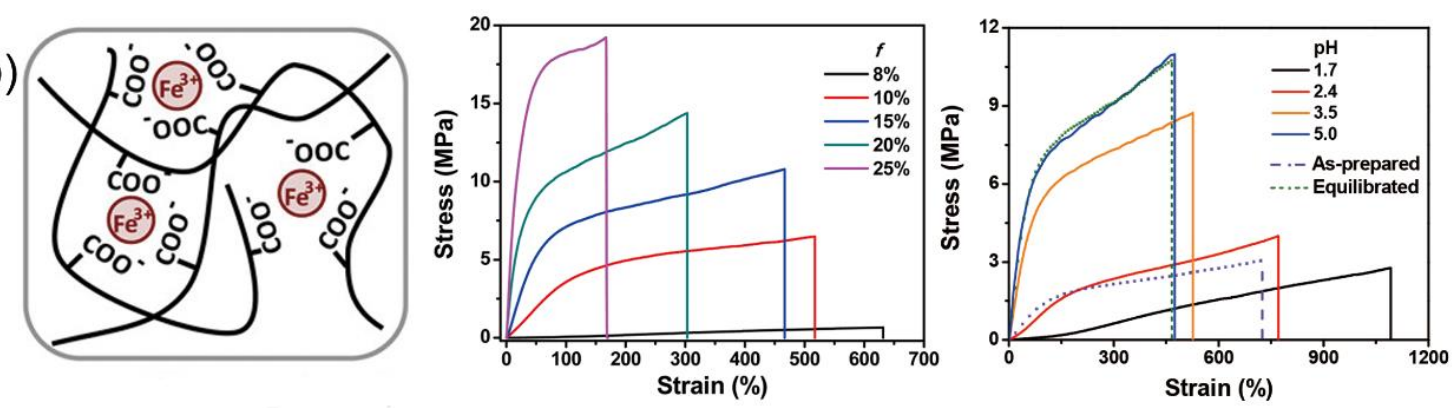

c)
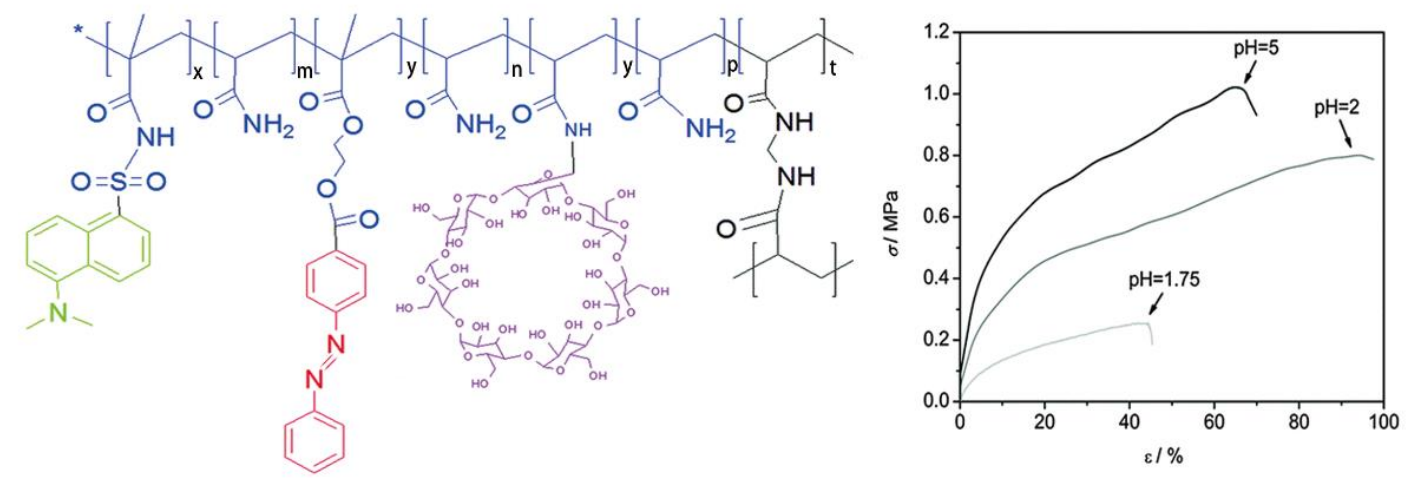

Figure 9. pH-responsive hydrogels with tunable mechanical properties. a) Schematic illustration of the state of hydrogen bonds in poly(1-vinylimidazole-co-acrylic acid) (P(VI-co-AAc)) hydrogel equilibrated in aqueous solutions of different $\mathrm{pH}$ and their corresponding mechanical properties. Reproduced with permission. ${ }^{148}$. Copyright 2017 Elsevier Ltd. b) Schematic representation of ligand-metal complexes formed uniaxial tensile properties of $\mathrm{Fe}^{3+}$ ions and poly(acrylic acid-coacrylamide) (P(AAm-co-AAc)-Fe ${ }^{3+}$ ) hydrogels with different feed ratios of AAc. The mechanical properties of hydrogels with AAc feed ratio of $15 \mathrm{~mol} \%$ incubated in solutions at different $\mathrm{pH}$ are shown on the right side. Reproduced from ${ }^{35}$ Copyright 2016 American Chemical Society. c) Chemical structure and uniaxial tensile properties of PAAm-based copolymeric hydrogels with dansyl side chains at different $\mathrm{pH}$. The $\mathrm{x}, \mathrm{y}$ and $\mathrm{t}$ are $10 \%, 6 \%, 10 \%$, respectively. Chemical 
870

871

872

873

874

875

876

877

878

879

880

881

882

883

884

885

886

887

888

structure slightly modified for better presenting the azobenzene group. Reproduced with permission. ${ }^{156}$ Copyright 2016 The Royal Society of Chemistry.

\section{Salt-responsive hydrogels}

In nature, inorganic ions are involved in numerous tasks. For instance, the influx of sodium ions into the cytoplasm and the diffusion of calcium ions into muscle fibers lead to the contraction of our muscles. Mimicking nature, hydrogels may undergo mechanical change when in the presence of inorganic salt. Indeed, the salt may play versatile functionalities in tuning hydrogels' rigidity, including affecting coordinate bonds, impacting polymer solubility, shielding ionic bonds, and controlling osmotic pressure.

\subsection{Influence of coordinate bonds}

In chemistry, coordinate bonds typically involve the binding of a metal to certain ligands such as carboxylate-ions to form coordination complexes. These coordination complexes are of both academic and practical interest. In the case of hydrogels having a certain amount of coordinate sites, the introduction of suitable transition metal ions can give rise to the formation of new crosslinking points and effectively affect the mechanical performance of the hydrogels. It should be noted that the toughening process induced by the formation of strong metal-coordinate bonds is limited to specific ion pairs, which can be generally explained by the classical hard-soft acid-base theory postulating that hard acids bind 
889 strongly to hard bases and soft acids strongly bind to soft bases. ${ }^{158}$

890 As a representative example, Chen et al. ${ }^{159}$ introduced $\mathrm{Fe}^{3+}$ into a double network gel 891 consisting of an agar gel, as the first physical network, embedded into a chemically 892 crosslinked second network of $\mathrm{P}(\mathrm{AAm}-\mathrm{co}-\mathrm{AAc})$ (PAMAAc). Besides the pH-tunable 893 performance derived from the AAc group, the gel demonstrates significant ion894 responsiveness. Compared to the gels without $\mathrm{Fe}^{3+}$, Agar/PAMAAc-Fe ${ }^{3+}$ gels demonstrate 895 an enhancement of fracture stress and hysteresis energy by 3.4 times and 2.8 times, 896 respectively (Figure 10a). The large amplitude of the hysteresis comes from the unzipping 897 of the additional physical network of coordinate complexes. ${ }^{160}$ Therefore, the formation of 898 reversible sacrificial ionic crosslinks appears as an interesting strategy to reinforce gel 899 properties by simply immersing polycarboxylic acid hydrogel into a suitable multivalent 900 salt solution. ${ }^{161}$

901 Metal ions can also significantly affect hydrogels' toughness by forming different dentate 902 ligands in coordination compounds; ${ }^{162}$ the mechanical properties being influenced by the 903 immersing time as well as the ion concentration. For example, poly(acrylonitrile-co-2904 methacryloyloxyethyl phosphorylcholine) (P(AN-co-MPC)) hydrogels, designed with 905 dipole-dipole interactions between cyano groups (CN-CN pairs) and coordinate bonds 906 between $\mathrm{CN}$ and $\mathrm{Zn}^{2+}$ ions, were developed by Han et al. ${ }^{34}$ As shown in Figure $10 \mathrm{~b}$, the 907 hydrogel, which is initially reinforced by the strong CN-CN interactions within the network, 
908 shrinks progressively when immersed in $\mathrm{ZnCl}_{2}$ solutions as zinc ions may serve as 909 additional physical crosslinks between cyano groups due to favorable formation of $910 \mathrm{Zn}(\mathrm{CN})_{2}$. Concurrently, the Young modulus increases from $7 \mathrm{MPa}$, initially in water, up to $91138 \mathrm{MPa}$ in $30 \% \mathrm{ZnCl}_{2}$ solution. Then, when the zinc ion concentration is increased beyond $91230 \%$, excessive zinc ions bind to single $\mathrm{CN}$ groups and shield other cyano groups from 913 forming dipole-dipole interactions. In this range of concentrations $\left(\left[\mathrm{ZnCl}_{2}\right]>38 \%\right)$, the gel 914 highly swells and its Young's modulus sharply decreases up to $0.05 \mathrm{MPa}$ in $50 \% \mathrm{ZnCl}_{2}$. 915 Therefore, by adjusting the concentration of $\mathrm{ZnCl}_{2}$ in the external solution, the authors 916 demonstrate that the elastic modulus of the gel can be tuned over a very wide range, from $917 \mathrm{kPa}$ to $\mathrm{MPa}$, allowing shape memory properties to be developed with various temporary 918 and permanent shapes. 
a)

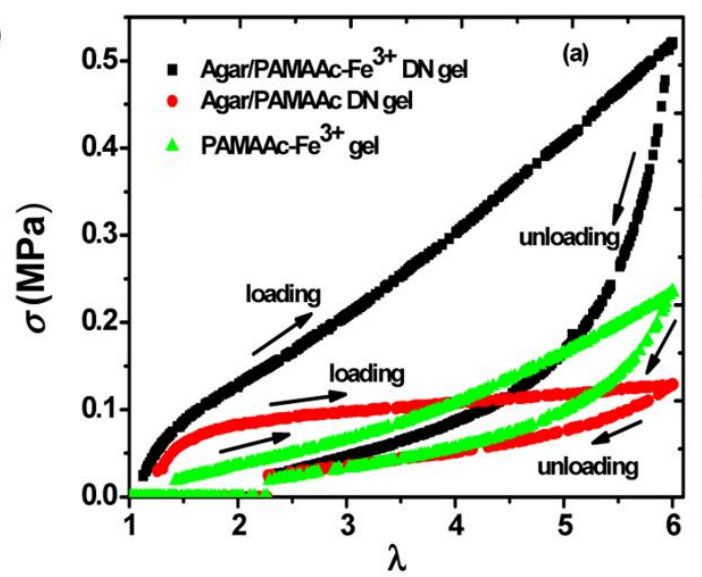

b)

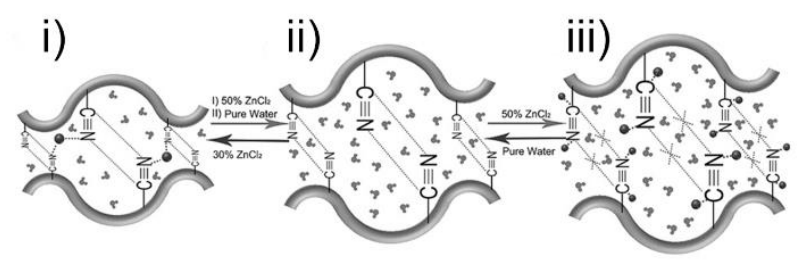
GmbH \& Co. KGaA, Weinheim.
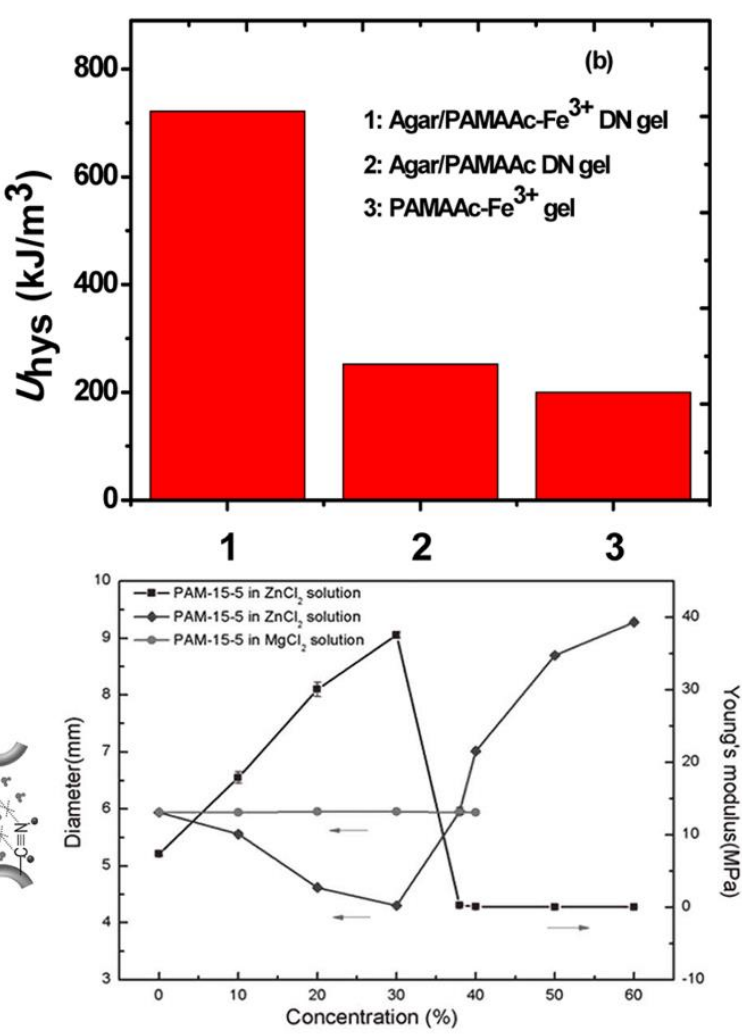

Figure 10. Mechanism and mechanical properties of hydrogels with salt-responsiveness. a) Loading-unloading curves and the corresponding dissipated energies of Agar/PAMAAc-Fe ${ }^{3+}$ DN gel, Agar/PAMAAc DN gel, and PAMAAc-Fe ${ }^{3+}$ gel. Reproduced from ${ }^{159}$ Copyright 2016 American Chemical Society. b) Schematic representation of 3 different hydrogel states featuring: i) $\mathrm{CN}-\mathrm{CN}$ $+\mathrm{CN}-\mathrm{Zn}$ interactions, ii) $\mathrm{CN}-\mathrm{CN}$ interactions and iii) dissociation of the previous interactions of poly(acrylonitrile 2-methacryloyloxyethyl phosphorylcholine) hydrogel (named PAM in the figure) in response to zinc ions. On the right side, the figure shows the variation of Young's modulus (square $\square$ ) and the diameter (diamond $\diamond)$ of the gel for different concentrations of $\mathbf{Z n C l}_{2}\left(\mathrm{MgCl}_{2}\right.$ solution serves as a control). Reproduced with permission. ${ }^{34}$ Copyright 2012 WILEY-VCH Verlag

Similar performances have been demonstrated in polycarboxylate systems with other metal 
932 ions including $\mathrm{Ca}^{2+},{ }^{152,154,161,163-164} \mathrm{Al}^{3+},{ }^{163}$ and $\mathrm{Cu}^{2+}$ ions ${ }^{152}$. Such behaviors are highly

933 related to the type of central metal and ionizable ligand, as the bond stability fundamentally

934 scales with the decreasing size of the cation. ${ }^{154}$ Moreover, $\mathrm{Zr}^{4+}$ ions which are known to

935 form coordinate bonds with sulfonate groups, can effectively reinforce such hydrogels. ${ }^{165}$

936 For instance, the immersion of a poly(acrylamide-co-2-acrylamido-2-methyl-1-

937 propanesulfonic acid) ( $\mathrm{P}(\mathrm{AAm}-\mathrm{co}$-AMPS) $)$ hydrogel into a $\mathrm{ZrOCl}_{2}$ solution gives rise to

938 an enhancement in fracture stress of 3 orders of magnitude (from $2.5 \mathrm{kPa}$ to $6.3 \mathrm{MPa}$ ).

939 Upon stretching, the sulfonate- $\mathrm{Zr}^{4+}$ coordination complexes can break, dissipating energy,

940 and reform, ensuring satisfactory mechanical performance. Owing to the dynamic nature

941 of the coordinate bonds, these hydrogels show rate- and temperature-dependent mechanical

942 performances, as well as good self-recovery properties. These hydrogels enable versatile

943 applications in the biomedical and engineering fields, such as artificial tubular grasper.

$944 \quad 5.2$ Solubility effect

945 Besides the aforementioned mechanisms, salt can also modify the characteristics of

946 hydrogels by affecting the polymer/water interactions. Historically, Hofmeister was the

947 first to report that the water solubility of proteins could be increased or decreased in a

948 controlled manner by adjusting the nature and concentration of the salt. Nowadays, the

949 ranking of the physical properties of ions in aqueous solutions, known as Hofmeister series,

950 is a benchmark widely used to modify the solubility of polymers and smaller molecules in 
951 water. ${ }^{166}$ Although the underlying mechanisms are still under debate after more than one 952 century, hydrogen bonding, dispersion interactions, hydration forces, or even dissolved gas 953 have been suggested for this phenomenon. ${ }^{167}$ In the case of PNIPAm gels, for which 954 interactions in water result from a compromise between hydrogen bonds and hydrophobic 955 interactions, the transition temperature as well as the macroscopic properties can be finely 956 tuned by applying the so-called Hofmeister effects. ${ }^{168}$

957 In the Hofmeister series, ions are divided into "kosmotropes" and "chaotropes" with 958 salting-out and salting-in effects, respectively, indicating how the interactions of small 959 solute molecules with water affect the solubility of large molecules. ${ }^{169}$ While the salting960 out effect tends to reduce the hydration of polymer chains, ${ }^{170}$ strengthen specific physical 961 interactions, ${ }^{171}$ and consequently reinforce the mechanical behavior of hydrogels, the 962 salting-in leads to opposite effects. In the work of Chen and co-authors, ${ }^{172}$ the mechanical 963 properties of poly(acrylamide)/methylcellulose (PAAm/MC) hydrogels were significantly 964 improved by simply soaking the gel in solutions of kosmotropic ions. By increasing the 965 hydrophobic interactions, the small kosmotropic anions thus led to chain bundling, 966 endowing the hydrogels with enhanced mechanical properties. For instance, the tensile 967 strength of the treated hydrogel reached as high as $4.4 \mathrm{MPa}$, which is more than 30 times 968 higher than that of the original hydrogel. Upon stretching, these hydrophobic associations 969 readily break, dissipating energy, and then reform guaranteeing good mechanical 970 performance. Following this strategy, the mechanical properties of these hydrogels can be 
971 further tuned by varying the post soaking time and the kind of Hofmeister salts. The 972 PAAm/MC hydrogels treated with solutions of strong "salting-out" anions like $\mathrm{Cit}^{3-}, \mathrm{CO}_{3}{ }^{2-}$ 973 and $\mathrm{SO}_{4}{ }^{2-}$, reached higher compression strength of 27.6, 19.6, and 14.2 MPa, respectively. 974 In comparison, the same gel immersed in a solution containing a weak "salting-out" anion $975\left(\mathrm{Cl}^{-}\right)$exhibits a much weaker fracture compression stress $(7.4 \mathrm{MPa})$. While the high 976 mechanical performance of this hydrogel is maintained in saline solution, the hydrogel 977 swells and loses its mechanical strength when immersed in pure water. ${ }^{172}$ Very recently, He 978 and coauthors attested that the mechanical properties of poly(vinyl alcohol) (PVA) 979 hydrogels can be well-tuned at a broad range by simply soaking the as-prepared gels in 980 different ions solutions. ${ }^{173}$ As shown in Figure 11a, hydrogen bonds forming or breaking 981 can be induced by ions due to the salting-out effect or salting-in effect. With different media 982 soaked, the PVA hydrogels demonstrate tensile strength from $50 \mathrm{kPa}$ to $15 \mathrm{MPa}$, toughness 983 from 0.0167 to $150 \mathrm{MJ} \mathrm{m}^{-3}$, and Young's modulus from 24 to $2500 \mathrm{kPa}$ (Figure $11 \mathrm{~b}$ ). It is 984 verified that the Hofmeister effect accounts for this phenomenon by affecting the 985 aggregation states of the polymer chains and the formation of structures, rather than just 986 affecting the hydration level or playing a role as the components of hydrogels. The 987 Hofmeister effect becomes more pronounced with crystalline hydrogels as the salt also 988 affect the crystalline degree in PVA hydrogels. ${ }^{174}$

989 The salt-sensitive mechanical response of hydrogels can also be finely adjusted by simply 990 controlling the immersion time. Cui et al. ${ }^{175}$ have developed chitosan/poly(acrylic acid) 
991 double network nanocomposite hydrogels (CS/PAA/TA@CNC) via in situ polymerization 992 of acrylic acid in chitosan acid aqueous solution with tannic acid-coated cellulose 993 nanocrystal acting as nanofillers. These nanocomposite hydrogels were then soaked into a 994 saturated $\mathrm{NaCl}$ solution in order to aggregate chitosan chains by shielding electrostatic 995 repulsions between positively charged amino groups. Compared with the original hydrogel, 996 without soaking treatment, the "CS/PAA/TA@CNC-60" gel, which was immersed for 60 $997 \mathrm{~min}$ in a saturated $\mathrm{NaCl}$ solution, exhibits a significant enhancement of the mechanical 998 properties with a 10 -fold increase in the fracture stress and a 2 -fold increase in the fracture 999 strain, which is attributed to a higher crosslinking density of chitosan induced by chain 1000 entanglement and salting-out effect (Figure 11c). With further extended soaking time, the 1001 fracture energy and elastic modulus began to decrease due to excessive shielded 1002 electrostatic repulsions, which has another salt effect will be discussed in the following 1003 part. 
a)

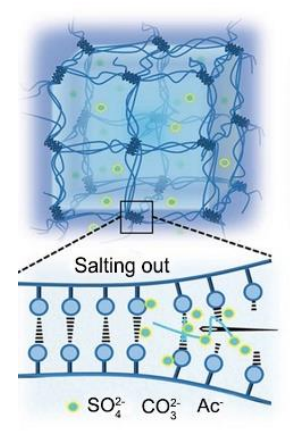

b)

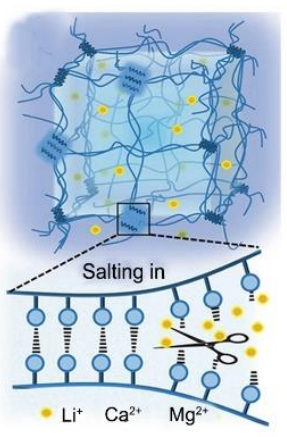

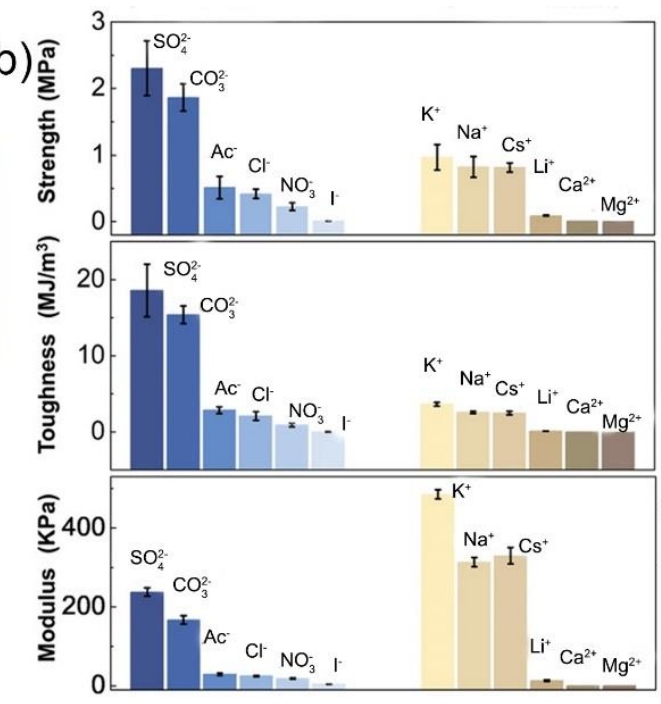

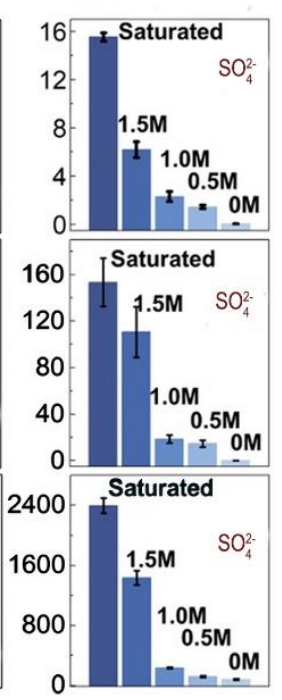

c)

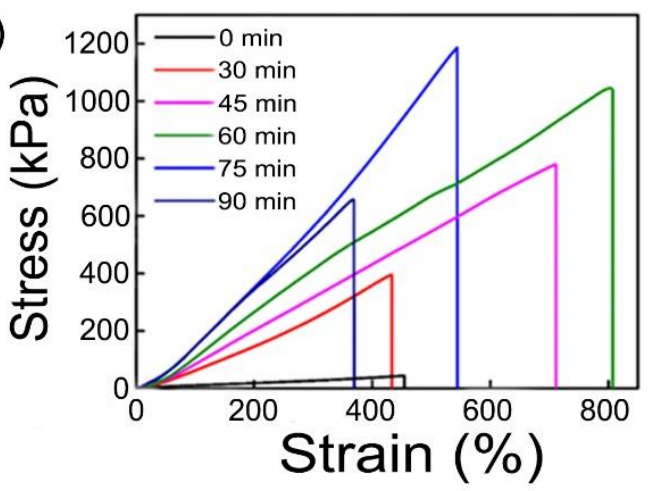

1004

1005

1006

1007

1008

1009

1010

1011

1012

1013

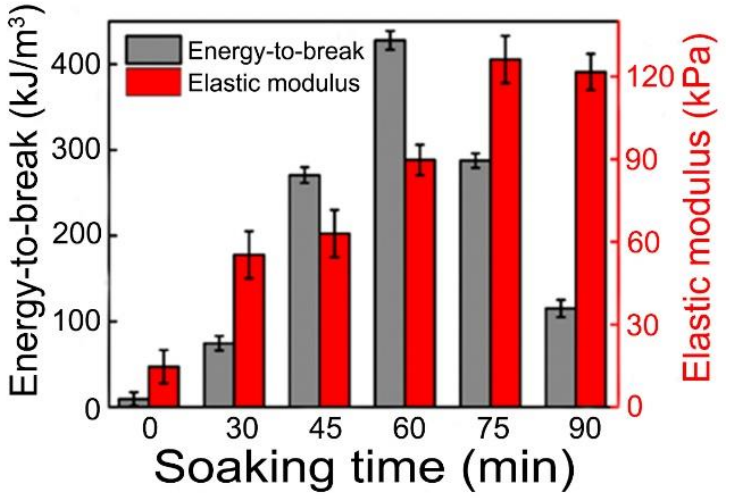

Figure 11. Mechanism and mechanical properties of hydrogels with Hofmeister effect. a) Hydrogen bonds form or break between PVA polymer chains induced by ions due to salting-out or salting-in effect. b) Strengths, toughness, and moduli of PVA hydrogels tuned by various anions (with $\mathrm{Na}^{+}$as the constant counterion); different cations (with $\mathrm{Cl}^{-}$as the constant counterion); and $\mathrm{Na}_{2} \mathrm{SO}_{4}$ with concentrations ranging from $\mathrm{O}$ m to saturated. Reproduced with permission. ${ }^{173}$ Copyright 2021 Wiley-VCH GmbH. c) Tensile stress-strain curves, elastic modulus, and energyto-break of the tannic acid-coated cellulose nanocrystal CS/PAA/TA@CNC DN nanocomposite hydrogels with different soaking time in saturated $\mathrm{NaCl}$ solution. Reproduced from ${ }^{175}$. Copyright 2019 American Chemical Society. 
$1015 \quad 5.3$ Shielding of ionic interactions

1016 In hydrogels, ionic interactions greatly contribute to strengthening their properties. Upon

1017 stretching, these noncovalent bonds can reversibly break to dissipate energy and reform to

1018 guarantee their mechanical performance and stability. Consequently, the addition of small

1019 molecular salts can effectively screen the attractive interactions between oppositely

1020 charged groups carried by macromolecular chains, leading to the release of entangled

1021 polymer chains. ${ }^{39,175}$

1022 Polyampholytes (PA) hydrogels, containing both anions and cations in their

1023 macromolecular structure, are typical examples of materials sensitive to salt additives due

1024 to electrostatic screening. In the pioneering work of Gong et al., ${ }^{176}$ PA hydrogels were

1025 prepared with an equal amount of the 3-(methacryloylamino) propyl-trimethylammonium

1026 chloride (MPTC, cationic monomer) and sodium p-styrenesulfonate (NaSS, anionic

1027 monomer). Close to the charge balance in pure water, these hydrogels display extremely

1028 high toughness with remarkable viscoelasticity which is attributed to the very wide

1029 distribution of ionic bond strengths. The strong bonds serve as permanent crosslinks,

1030 imparting elasticity, while the weak bonds reversibly break and reform, dissipating energy.

1031 However, the addition of inorganic salt gradually weakens the strength of electrostatic

1032 interactions and leads to a significant decrease in Young's modulus (from 2.2 to 0.1 MPa)

1033 and fracture stress $\sigma_{b}$ (from 2.60 to $0.07 \mathrm{MPa}$ ). Unlike the intrachain ionic complexation

1034 that takes place in PA hydrogels, polyion complex (PIC) hydrogels prepared from equal 
oppositely charged homo-polyelectrolytes can only lead to interchain complexation

1036 between polymers in the extended coil conformation. This interchain complexation, which

1037 takes place even at low polymer concentrations, greatly promotes the chain entanglement

1038 and the stability of ionic complexes, thus making the mechanical properties much superior

1039 to those of PA hydrogels with the same rate of combination of monomers. When these PIC

1040 hydrogels synthesized from MPTC and NaSS homopolymers are immersed in saline

1041 solutions of increasing concentration, a decrease in the breaking stress as well as a

1042 significant increase in fracture strain are observed (Figure 12a). ${ }^{177}$ By tuning the screening

1043 of interpolymer electrostatic interactions with salt, it is possible to control the dynamics of

1044 ionic bonds and endow the hydrogel with self-healing properties. For instance, after

1045 soaking two pieces of hydrogels for 2 minutes in $3 \mathrm{M} \mathrm{NaCl}$ and bringing them into contact

1046 for 12 hours at room temperature, the self-healed sample exhibits a recovery rate of $66 \%$

1047 in terms of work of extension with high strength (modulus $\sim 4.5 \mathrm{MPa}$; fracture stress $\sim 2.2$

$1048 \mathrm{MPa}$; fracture strain $\sim 630 \%$ ).

$1049 \quad 5.4$ Controlling osmotic pressure

1050 In hydrogels, water plasticizes polymers by disrupting chain interactions, creating free

1051 volume in the polymer network, enhancing chain mobility, and softening the material. The

1052 ionic contribution to the osmotic pressure has a huge effect on the swelling properties of

1053 gels and it can be used either to generate super swelling properties, as in superabsorbent

1054 polymers or to cause the gel collapsing when the excess of ionic pressure inside the network 
1055 is counterbalanced by that of the external environment with the addition of salt. Such 1056 osmotic deswelling, which increases the density of polymer chains in the network, also 1057 participates in strengthening the specific physical interactions.

1058 This phenomenon has been particularly investigated in ionic gels using additional 1059 noncovalent associations as nicely exemplified by Gong and coworkers who couple 1060 electrostatic and hydrophobic interactions within the same network. ${ }^{178}$. As shown in Figure $106112 b$, the authors synthesized copolymers through cation- $\pi$ complex-aided free-radical 1062 polymerization between an aromatic monomer and a cationic one. While the copolymer 1063 itself is water-soluble, due to electrostatic repulsions between the chains dominating the 1064 solution behavior, the addition of salt favors the formation of a gel coacervate as salt ions 1065 screen long-range electrostatic repulsions and strengthen the effective cation- $\pi$ and 1066 hydrophobic interactions taking place intra- and inter-molecularly (Figure 12c). While the 1067 copolymer solution prepared in pure water is not sufficiently elastic to be able to withstand 1068 deformation, the system becomes much harder and more stretchable in salty environments 1069 (Figure 12d). Such materials, which are also characterized by self-healing and adhesive 1070 properties in saline conditions, are promising systems paving the way for the development 1071 of responsive hydrogels in physiological and marine environments. 
a)

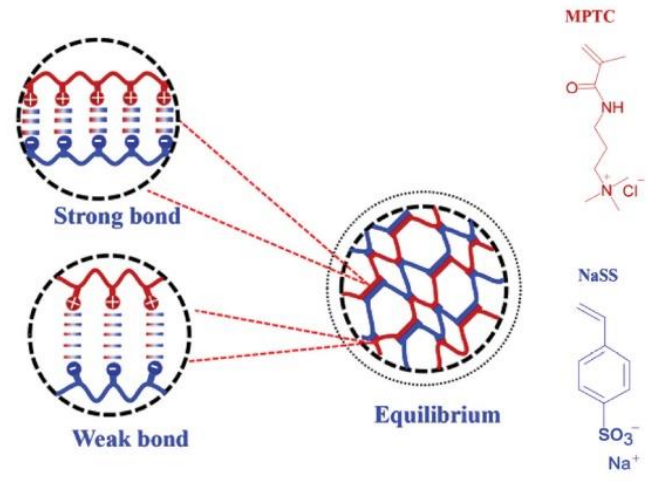

b)

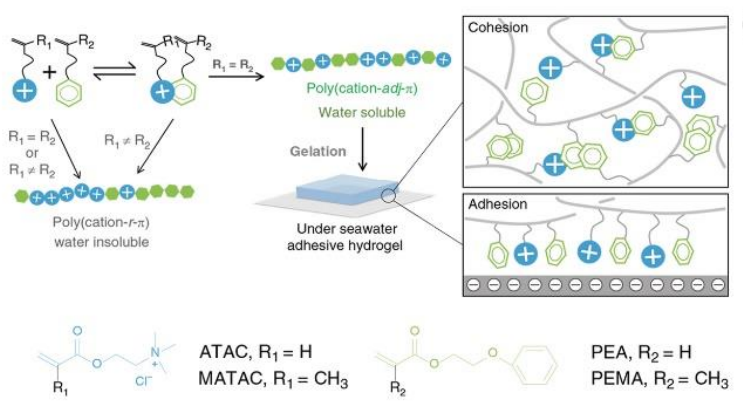

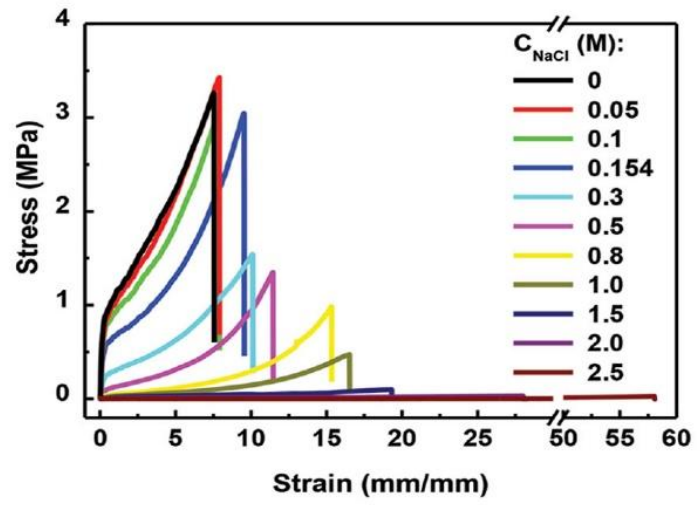

c)

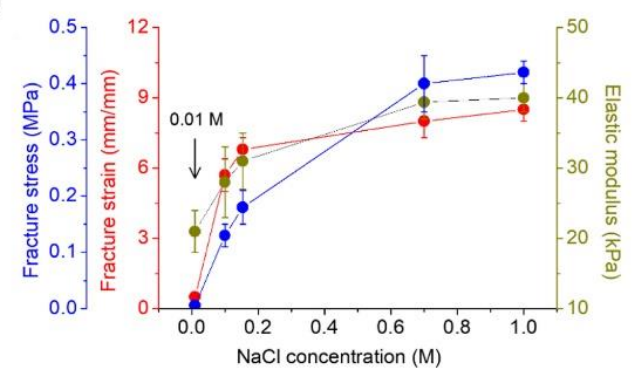

d)

1072

1073

1074

1075

1076

1077

1078

1079

1080

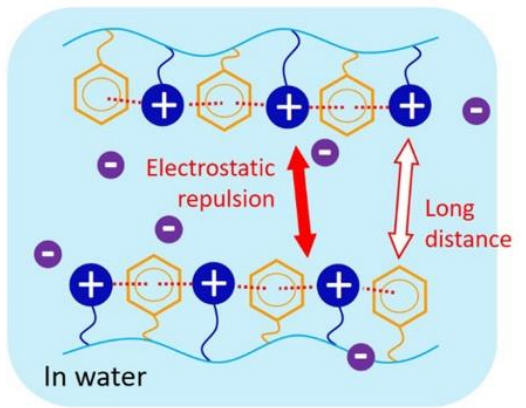

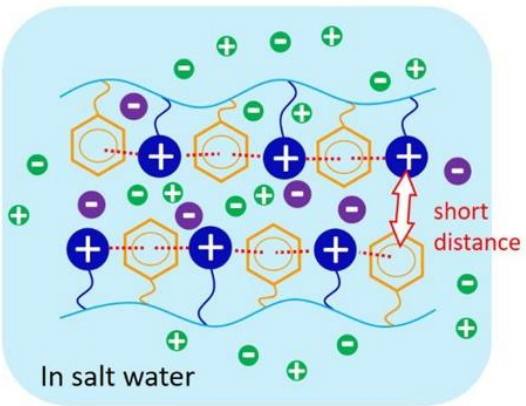

Figure 12. Salt-sensitive hydrogels with shielding of ionic interactions and controlling osmotic pressure. a) Schematic illustration of PIC hydrogels prepared from poly(3-(methacryloylamino) propyl-trimethylammonium chloride) (MPTC) and poly(sodium p-styrenesulfonate) (NaSS) and uniaxial performance in response to immersion in different $\mathrm{NaCl}$ solutions. The chemical structure of corresponding monomers is illustrated on the left side. Reproduced with permission. ${ }^{177}$ Copyright 2015 WILEY-VCH Verlag GmbH \& Co. KGaA, Weinheim. b) Schematic illustration of design strategy with chemical structures of typical monomers used, c) fracture stress, fracture strain and elastic modulus obtained from stress-strain curves with 
1084 As already discussed, the introduction of salt which will favor the formation of physical1085 crosslinked domains largely contributes to the energy dissipation and endow the gels with 1086 reinforced mechanical performance. ${ }^{179}$ Using the simple soaking process, salt-responsive 1087 hydrogels prepared from natural or synthetic macromolecules can be easily strengthened

1088 for use in versatile applications, such as bone regeneration, ${ }^{33}$ tubular graspers, ${ }^{165}$ sensors..${ }^{175}$

1089 Nevertheless, as discussed in the case of $\mathrm{pH}$-sensitive hydrogels, the time required for the 1090 solutes to diffuse within the gel can be a significant obstacle for some practical applications.

\section{Conclusion and perspective}

1092 Over the past 4 decades, hydrogels with responsive properties have been definitively a hot 1093 topic with significant academic developments. Until the 1990s, researchers mainly focused 1094 on the thermodynamic aspects of the volume phase transition of hydrogels in response to 1095 environmental stimuli, and many new and complex architectures have been designed and 1096 studied within this framework. Since the beginning of the new century, the vast knowledge 1097 acquired on these systems, as well as the development of experimental techniques, have 1098 led to direct research towards the mechanical properties of hydrogels in order to elaborate 1099 smart and efficient reinforcement strategies. This new orientation, stimulated in particular 1100 by the potential of these swollen materials in biomedical applications, has led to a 
1101 multiplication and a sophistication of the systems studied, making it possible to consider

1102 the control of their mechanical properties using single or multiple environmental triggers.

1103 Today, there is a truly solid basis of knowledge for the structure/properties relationships of

1104 smart hydrogels characterized by responsive mechanical properties under thermal, light,

$1105 \mathrm{pH}$, and/or salt triggers, but improvements are needed and we have still a lot to learn from

1106 nature.

1107 In the case of stimuli-responsive gels, not only thermodynamics prevails but also kinetic

1108 aspects play an important role at different scales that must be considered in the transition

1109 process. On the one hand, the rate of thermal transfer is faster compared to the other

1110 processes (e.g. 2-3.10 $0^{-3} \mathrm{~cm}^{2} \cdot \mathrm{s}^{-1}$. for PNIPAm). On the other hand, the transport rate of

1111 solvent or solute into or out of the network is much slower. In the case of a purely diffusive

1112 mechanism, the experimental values measured in the gels are close to the self-diffusion

1113 coefficients of the molecules (e.g. 2.6.10-5 $\mathrm{cm}^{2} \cdot \mathrm{s}^{-1}$ for $\mathrm{D}_{2} \mathrm{O}$ ). Moreover, the movement of

1114 polymer chains further limits the kinetic process in the case of large volume phase

1115 transition. On average, the collective diffusion coefficient for a homogeneous gel is around

11162 orders of magnitude lower compared to the solvent and 4 orders compared to the thermal

1117 stimulus. In particular, this factor will be the limiting step of the kinetic process in the case

1118 of large volume phase transition, keeping in mind that the characteristic swelling time $(\tau)$

1119 scales with the square of the size of the sample $(r)$ as: $\tau \sim r^{2} / D_{0}$. Such kinetic issues can

1120 be critical in the case of $\mathrm{pH}$ and/or salt responsive toughening, where environmental 
1121 modifications should be driven by soaking the gel in an external medium and waiting for

1122 minutes or even hours depending on the size of the sample. This is rather long compared

1123 to hydrogels found in the human body that display much shorter characteristic times on the

1124 order of a second or less. Photo-stimulation is an alternative a good way to overcome the

1125 problems associated with the slow diffusion of solutes into the gel, but the short

1126 penetrability of light remains a critical issue. Another problem that deserves to be pointed

1127 out concerns the sensitivity of the gel to a stimulus. Indeed, while in nature an infinitesimal

1128 change in biological signals can induce a significant mechanical response, synthetic

1129 responsive hydrogels require a greater variation in environmental conditions to provide a

1130 macroscopic response. In addition, the lack of reversibility of some responsive hydrogels

1131 also limits their long-term use.

1132 With an increasing understanding of the structure/property relationships of these soft and

1133 wet materials, new versatile systems will certainly emerge in the future. On the one hand,

1134 an increasing number of smart hydrogels whose mechanical performances are responsive

1135 to novel stimuli may come on the scene, e.g. the electrical, magnetic, touch, or

1136 electrochemical triggers. These input signals are ubiquitous in bio-tissues, such as our

1137 central nervous system takes the art of electrochemistry for nerve conduction and muscle

1138 movement control. Different from currently applied triggers, these new stimuli may safely

1139 overcome the existing problem of low responsive time. Although hydrogels with such

1140 stimuli become increasingly valued in recent years, the related researches are still in their 
1141 infancy compared to the real cases in nature. On the other hand, new hydrogels with

1142 stimuli-responsive mechanical performance may appear with a super-low threshold value.

1143 Furthermore, the gels may even become capable to differentiate between similar inputs.

1144 These perspectives are highly challenging for existing hydrogels but out of question in the

1145 biological world. For example, the motion of our smooth muscle is closely associated with

1146 very tiny environmental $\mathrm{pH}$ variation and the physiological effect of sodium and potassium

1147 ions are poles apart. Another important issue is how to create hydrogels with step-by-step

1148 regulation in mechanical performance. Taking the Mimosa Pudica for illustration: upon

1149 touching, the plant experiences stimulus perception at first, giving rise to electrical signal

1150 transmission in the next and finally induces rapid mechanical movement. The more intense

1151 the stimuli, the longer it takes to maintain the closing state. The advantage of this cascade

1152 system is that it is able to produce fast, delicate, adaptive, and reversible mechanical output.

1153 Nevertheless, there is still a certain distance before obtaining this kind of artificial soft

1154 matter.

1155 Indeed, after 40 years of intensive studies on responsive hydrogels, we have learned a lot 1156 about how to use physical interactions to control the macroscopic response of the hydrogel

1157 and in particular how to modulate the mechanical properties. Although we still have a long 1158 way to go by learning from nature, the weaknesses of current hydrogels will be the next 1159 milestones for future research with an increasing focus on practical applications in the near 1160 future. 
1161 AUTHOR INFORMATION

1162 Corresponding authors

1163 E-mail: guoh37@ mail.sysu.edu.cn (H. G.);

1164 E-mail: dominique.hourdet@espci.fr (D. H.)

1165 ORCID:

1166 Xiaolin Wang: 0000-0002-2870-1111

1167 Zi Liang Wu: 0000-0002-1824-9563

1168 Hui Guo: 0000-0002-4652-2395

1169 Dominique Hourdet: 0000-0002-0328-7014

1170 Author Contributions

1171 These authors contribute equally to this work.

1172 Notes

1173 The authors declare no conflict of interest.

1174

1175 ACKNOWLEDGMENT

1176 The authors gratefully acknowledge the financial support from the National Natural

1177 Science Foundation of China (NSFC) (No. 51903253), Natural Science Foundation of

1178 Guangdong Province of China (2019A1515011150, 2019A1515011258), and the Science

1179 and Technology Development Fund of Macao (FDCT 0009/2019/A, 0083/2019/A2, $1180 \quad 0007 / 2019 / \mathrm{AKP})$. 
1182 (1) Montero de Espinosa, L.; Meesorn, W.; Moatsou, D.; Weder, C. Bioinspired Polymer 1183 Systems with Stimuli-Responsive Mechanical Properties. Chem. Rev. 2017, 117, 12851118412892.

1185 (2) Zhang, D.; Ren, B.; Zhang, Y.; Xu, L.; Huang, Q.; He, Y.; Li, X.; Wu, J.; Yang, J.; Chen, 1186 Q.; Chang, Y.; Zheng, J. From design to applications of stimuli-responsive hydrogel strain 1187 sensors. J. Mater. Chem. B 2020, 8, 3171-3191.

1188 (3) Ebara, M.; Kotsuchibashi, Y.; Uto, K.; Aoyagi, T.; Kim, Y.-J.; Narain, R.; Idota, N.; 1189 Hoffman, J. M. Smart Hydrogels. In Smart Biomaterials; Springer: Tokyo, 2014; pp 9-65.

1190 (4) Vijay Kumar Thakur, M. K. T. Hydrogels. In Gels Horizons: From Science to Smart 1191 Materials; Vijay Kumar Thakur, M. K. T., Ed.; Springer Singapore: Singapore, 2018.

1192 (5) Wang, X.; Ronsin, O.; Gravez, B.; Farman, N.; Baumberger, T.; Jaisser, F.; Coradin, T.; 1193 Hélary, C. Nanostructured Dense Collagen-Polyester Composite Hydrogels as Amphiphilic 1194 Platforms for Drug Delivery. Adv. Sci. 2021, 8, 2004213.

1195 (6) Qiu, Y.; Park, K. Environment-sensitive hydrogels for drug delivery. Adv. Drug Deliv. 1196 Rev. 2012, 64, 49-60.

1197 (7) Yang, C.; Suo, Z. Hydrogel ionotronics. Nat. Rev. Mater. 2018, 3, 125-142.

1198 (8) Yuk, H.; Lu, B.; Zhao, X. Hydrogel bioelectronics. Chem. Soc. Rev. 2019, 48, 164211991667.

1200 (9) Qin, H.; Zhang, T.; Li, N.; Cong, H. P.; Yu, S. H. Anisotropic and self-healing hydrogels 1201 with multi-responsive actuating capability. Nat. Commun. 2019, 10, 2202.

1207 (12) Liu, X.; Liu, J.; Lin, S.; Zhao, X. Hydrogel Machines. Mater. Today 2020, 36, 1021208124.

1209 (13) Buwalda, S. J.; Boere, K. W. M.; Dijkstra, P. J.; Feijen, J.; Vermonden, T.; Hennink, 1210 W. E. Hydrogels in a historical perspective: From simple networks to smart materials. $J$. 1211 Control. Release 2014, 190, 254-273.

1212 (14) Shintake, J.; Cacucciolo, V.; Floreano, D.; Shea, H. Soft Robotic Grippers. Adv. Mater. 1213 2018, 30, 1707035.

1214 (15) Shi, Q.; Liu, H.; Tang, D.; Li, Y.; Li, X.; Xu, F. Bioactuators based on stimulus1215 responsive hydrogels and their emerging biomedical applications. NPG Asia Mater. 2019, $1216 \quad 11,64$. 
1217 (16) Zhou, Y.; Dong, X.; Mi, Y.; Fan, F.; Xu, Q.; Zhao, H.; Wang, S.; Long, Y. Hydrogel 1218 smart windows. J. Mater. Chem. A 2020, 8, 10007-10025.

1219 (17) Sneddon, I. N.; Berry, D. S. The Classical Theory of Elasticity. In Elasticity and 1220 Plasticity / Elastizität und Plastizität; Flügge, S., Ed.; Springer Berlin Heidelberg: Berlin, 1221 Heidelberg, 1958; pp 1-126.

1222 (18) Obukhov, S. P.; Rubinstein, M.; Colby, R. H. Network Modulus and Superelasticity. 1223 Macromolecules 1994, 27, 3191-3198.

1224 (19) Schröder, U. P.; Oppermann, W. Mechanical and stress-optical properties of strongly 1225

1226 (20) Rubinstein, M.; Colby, R. H.; Dobrynin, A. V.; Joanny, J.-F. Elastic Modulus and 1227 Equilibrium Swelling of Polyelectrolyte Gels. Macromolecules 1996, 29, 398-406.

1228 (21) Maugis, D.; Barquins, M. Fracture mechanics and the adherence of viscoelastic bodies. 1229 J. Phys. D: Appl. Phys. 1978, 11, 1989-2023.

1230 (22) Lake, G. J.; Lindley, P. B. The mechanical fatigue limit for rubber. J. Appl. Polym. Sci. 1231 1965, 9, 1233-1251.

1232 (23) Lake, G. J.; Lindley, P. B. Cut growth and fatigue of rubbers. II. Experiments on a 1233 noncrystallizing rubber. J. Appl. Polym. Sci. 1964, 8, 707-721.

1234 (24) Creton, C. 50th Anniversary Perspective: Networks and Gels: Soft but Dynamic and 1235 Tough. Macromolecules 2017, 50, 8297-8316.

1236 (25) Baumberger, T.; Caroli, C.; Martina, D. Fracture of a biopolymer gel as a viscoplastic 1237

1238 (26) Haque, M. A.; Kurokawa, T.; Kamita, G.; Gong, J. P. Lamellar Bilayers as Reversible 1239 Sacrificial Bonds To Toughen Hydrogel: Hysteresis, Self-Recovery, Fatigue Resistance, 1240 and Crack Blunting. Macromolecules 2011, 44, 8916-8924.

1241 (27) Zhao, X. Multi-scale multi-mechanism design of tough hydrogels: building dissipation 1242 into stretchy networks. Soft Matter 2014, 10, 672-87.

1243 (28) Bilici, C.; Ide, S.; Okay, O. Yielding Behavior of Tough Semicrystalline Hydrogels. 1244 Macromolecules 2017, 50, 3647-3654.

1245 (29) Guo, M.; Pitet, L. M.; Wyss, H. M.; Vos, M.; Dankers, P. Y.; Meijer, E. W. Tough 1246 stimuli-responsive supramolecular hydrogels with hydrogen-bonding network junctions. $J$. 1247 Am. Chem. Soc. 2014, 136, 6969-6977.

1248 (30) Nonoyama, T.; Lee, Y. W.; Ota, K.; Fujioka, K.; Hong, W.; Gong, J. P. Instant Thermal 1249 Switching from Soft Hydrogel to Rigid Plastics Inspired by Thermophile Proteins. Adv. 1250 Mater. 2020, 32, 1905878.

1251 (31) Nakajima, T. Generalization of the sacrificial bond principle for gel and elastomer 1252 toughening. Polym. J. 2017, 49, 477-485.

1253 (32) Hao, J.; Weiss, R. A. Viscoelastic and Mechanical Behavior of Hydrophobically 
Modified Hydrogels. Macromolecules 2011, 44, 9390-9398.

(33) Jiang, L. B.; Su, D. H.; Ding, S. L.; Zhang, Q. C.; Li, Z. F.; Chen, F. C.; Ding, W.; Zhang, S. T.; Dong, J. Salt - Assisted Toughening of Protein Hydrogel with Controlled Degradation for Bone Regeneration. Adv. Funct. Mater. 2019, 29, 1901314.

(34) Han, Y.; Bai, T.; Liu, Y.; Zhai, X.; Liu, W. Zinc ion uniquely induced triple shape memory effect of dipole-dipole reinforced ultra-high strength hydrogels. Macromol. Rapid Commun. 2012, 33, 225-231.

(35) Zheng, S. Y.; Ding, H.; Qian, J.; Yin, J.; Wu, Z. L.; Song, Y.; Zheng, Q. MetalCoordination Complexes Mediated Physical Hydrogels with High Toughness, Stick-Slip Tearing Behavior, and Good Processability. Macromolecules 2016, 49, 9637-9646.

(36) Yang, P.; Wang, X.; Fan, H.; Gu, Y. Effect of hydrogen bonds on the modulus of bulk polybenzoxazines in the glassy state. Phys. Chem. Chem. Phys. 2013, 15, 15333-15338.

(37) Holten-Andersen, N.; Harrington, M. J.; Birkedal, H.; Lee, B. P.; Messersmith, P. B.; Lee, K. Y.; Waite, J. H. pH-induced metal-ligand cross-links inspired by mussel yield selfhealing polymer networks with near-covalent elastic moduli. Proc. Natl. Acad. Sci. U. S. A. 2011, 108, 2651-2655.

(38) Fullenkamp, D. E.; He, L.; Barrett, D. G.; Burghardt, W. R.; Messersmith, P. B. Mussel-inspired histidine-based transient network metal coordination hydrogels. Macromolecules 2013, 46, 1167-1174.

(39) Sun, T. L.; Luo, F.; Kurokawa, T.; Karobi, S. N.; Nakajima, T.; Gong, J. P. Molecular structure of self-healing polyampholyte hydrogels analyzed from tensile behaviors. Soft Matter 2015, 11, 9355-9366.

(40) Rosales, A. M.; Vega, S. L.; DelRio, F. W.; Burdick, J. A.; Anseth, K. S. Hydrogels with Reversible Mechanics to Probe Dynamic Cell Microenvironments. Angew. Chem. Int. Ed. 2017, 56, 12132-12136.

(41) Liu, A.; Gao, X.; Xie, X.; Ma, W.; Xie, M.; Sun, R. Stiffness switchable supramolecular hydrogels by photo-regulating crosslinking status. Dyes Pigm. 2020, 177, 108288.

(42) Gandavarapu, N. R.; Azagarsamy, M. A.; Anseth, K. S. Photo-Click Living Strategy for Controlled, Reversible Exchange of Biochemical Ligands. Adv. Mater. 2014, 26, 2521 2526.

(43) Li, L.; Scheiger, J. M.; Levkin, P. A. Design and Applications of Photoresponsive Hydrogels. Adv. Mater. 2019, 31, 1807333.

(44) Iwaso, K.; Takashima, Y.; Harada, A. Fast response dry-type artificial molecular muscles with [c2] daisy chains. Nat. Chem. 2016, 8, 625-632.

(45) Liu, G.; Yuan, Q.; Hollett, G.; Zhao, W.; Kang, Y.; Wu, J. Cyclodextrin-based hostguest supramolecular hydrogel and its application in biomedical fields. Polym. Chem. 2018, 9, 3436-3449. 
1292 (46) Lake, G.; Thomas, A. The Strength of Highly Elastic Materials. Proc. Math. Phys. 1293 Eng. Sci. 1967, 300, 108-119.

1294 (47) English, A. E.; Mafé, S.; Manzanares, J. A.; Yu, X.; Grosberg, A. Y.; Tanaka, T. 1295 Equilibrium swelling properties of polyampholytic hydrogels. J. Chem. Phys. 1996, 104, $1296 \quad 8713-8720$.

1297 (48) Sato Matsuo, E.; Tanaka, T. Kinetics of discontinuous volume-phase transition of gels. 1298 J.Chem. Phys. 1988, 89, 1695-1703.

1299 (49) Cheng, S. Z. D. Chapter 1 - Introduction. In Phase Transitions in Polymers; Cheng, S. 1300 Z. D., Ed.; Elsevier: Amsterdam, 2008; pp 1-15.

1301 (50) Shibayama, M.; Tanaka, T. Volume phase transition and related phenomena of polymer 1302 gels. In Responsive Gels: Volume Transitions I; Dušek, K., Ed.; Springer Berlin Heidelberg: 1303 Berlin, Heidelberg, 1993; pp 1-62.

1304 (51) Sato, K.; Nakajima, T.; Hisamatsu, T.; Nonoyama, T.; Kurokawa, T.; Gong, J. P. Phase1305 Separation-Induced Anomalous Stiffening, Toughening, and Self-Healing of 1306 Polyacrylamide Gels. Adv. Mater. 2015, 27, 6990-6998.

1307 (52) Tang, J. D.; Li, C. H.; Li, H. M.; Lv, Z. Y.; Sheng, H.; Lu, T. Q.; Wang, T. J. Phase1308 separation induced extraordinary toughening of magnetic hydrogels. J. Appl. Phys. 2018, $1309123,185105$.

1310 (53) Liu, Q.; Li, S.; Zhang, P.; Lan, Y.; Lu, M. Facile preparation of PNIPAM gel with 1311 1312 improved deswelling kinetics by using 1-dodecanethiol as chain transfer agent. J. Polym. Res. 2007, 14, 397-400.

1319 (56) C J Whiting, A. M. V., P D Olmsted, T C B McLeish. Shear modulus of polyelectrolyte 1320 (54) Guo, H.; Nakajima, T.; Hourdet, D.; Marcellan, A.; Creton, C.; Hong, W.; Kurokawa, T.; Gong, J. P. Hydrophobic Hydrogels with Fruit-Like Structure and Functions. Adv. Mater. 2019, 31, 1900702.

(55) Mitsumata, T.; Honda, A.; Kanazawa, H.; Kawai, M. Magnetically tunable elasticity for magnetic hydrogels consisting of carrageenan and carbonyl iron particles. J. Phys. Chem. B 2012, 116, 12341-8.

1321 gels under electric field. J. Phys.: Condens. Matter 2001, 13, 1381-1393.

(57) Tungkavet, T.; Pattavarakorn, D.; Sirivat, A. Bio-compatible gelatins (Ala-Gly-ProArg-Gly-Glu-4Hyp-Gly-Pro-) and electromechanical properties: effects of temperature and electric field. J. Polym. Res. 2011, 19, 9759.

1324 (58) Hirotsu, S. Electric-Field-Induced Phase Transition in Polymer. Jpn. J. Appl. Phys. 1325 1985, 24, 396-388.

1326 (59) Boustta, M.; Colombo, P. E.; Lenglet, S.; Poujol, S.; Vert, M. Versatile UCST-based 1327 1328 thermoresponsive hydrogels for loco-regional sustained drug delivery. J. Control. Release 2014, 174, 1-6.

1329 (60) Aseyev, V.; Tenhu, H.; Winnik, F. Non-ionic Thermoresponsive Polymers in Water. In 
1330 Self Organized Nanostructures of Amphiphilic Block Copolymers II; Müller, A. H. E.; 1331 Borisov, O., Eds.; Springer Berlin Heidelberg: 2011; Chapter 57, pp 29-89.

1332 (61) Scarpa, J. S.; Mueller, D. D.; Klotz, I. M. Slow hydrogen-deuterium exchange in a 1333 non-.alpha.-helical polyamide. J. Am. Chem. Soc. 1967, 89, 6024-6030.

(62) Lutz, J.-F.; Akdemir, Ö.; Hoth, A. Point by Point Comparison of Two Thermosensitive Polymers Exhibiting a Similar LCST: Is the Age of Poly(NIPAM) Over? J. Am. Chem. Soc. 2006, 128, 13046-13047.

1337 (63) Takigawa, T.; Yamawaki, T.; Takahashi, K.; Masuda, T. Change in Young's modulus 1338 of poly(N-isopropylacrylamide) gels by volume phase transition. Polymer Gels and 1339 Networks 1998, 5, 585-589.

1340 (64) Puleo, G. L.; Zulli, F.; Piovanelli, M.; Giordano, M.; Mazzolai, B.; Beccai, L.; 1341 Andreozzi, L. Mechanical and rheological behavior of pNIPAAM crosslinked 1342 macrohydrogel. React. Funct. Polym. 2013, 73, 1306-1318.

1343 (65) Gundogan, N.; Melekaslan, D.; Okay, O. Rubber elasticity of poly(N1344 isopropylacrylamide) gels at various charge densities. Macromolecules 2002, 35, 561613455622.

1346 (66) Matzelle, T. R.; Geuskens, G.; Kruse, N. Elastic Properties of Poly(N1347 isopropylacrylamide) and Poly(acrylamide) Hydrogels Studied by Scanning Force 1348 Microscopy. Macromolecules 2003, 36, 2926-2931.

1349 (67) Dragan, E. S. Design and applications of interpenetrating polymer network hydrogels. 1350 A review. Chem. Eng. J. 2014, 243, 572-590.

1351 (68) Li, Z.; Shen, J.; Ma, H.; Lu, X.; Shi, M.; Li, N.; Ye, M. Preparation and characterization 1352 of $\mathrm{pH}$ - and temperature-responsive nanocomposite double network hydrogels. Mater. Sci. 1353 Eng. C 2013, 33, 1951-1957.

1354 (69) Fei, R.; George, J. T.; Park, J.; Grunlan, M. A. Thermoresponsive nanocomposite 1355

1356 (70) Fei, R.; George, J. T.; Park, J.; Means, A. K.; Grunlan, M. A. Ultra-strong 1357 thermoresponsive double network hydrogels. Soft Matter 2013, 9, 2912.

1358 (71) Zheng, W. J.; An, N.; Yang, J. H.; Zhou, J.; Chen, Y. M. Tough Al-alginate/Poly(N1359 isopropylacrylamide) Hydrogel with Tunable LCST for Soft Robotics. ACS Appl. Mater. 1360 Interfaces 2015, 7, 1758-1764.

1361 (72) Haraguchi, K.; Takehisa, T.; Fan, S. Effects of clay content on the properties of 1362 nanocomposite hydrogels composed of poly(N-isopropylacrylamide) and clay. 1363

1364 (73) Du, H.; Wickramasinghe, R.; Qian, X. Effects of salt on the lower critical solution 1365 temperature of poly (N-isopropylacrylamide). J. Phys. Chem. B 2010, 114, 16594-16604.

1366 (74) Guo, H.; Brûlet, A.; Rajamohanan, P. R.; Marcellan, A.; Sanson, N.; Hourdet, D. 1367 Influence of topology of LCST-based graft copolymers on responsive assembling in 
aqueous media. Polymer 2015, 60, 164-175.

1369 (75) Halperin, A.; Kröger, M.; Winnik, F. M. Poly(N-isopropylacrylamide) Phase 1370 Diagrams: Fifty Years of Research. Angew. Chem. Int. Ed. 2015, 54, 15342-15367.

1371 (76) Haq, M. A.; Su, Y.; Wang, D. Mechanical properties of PNIPAM based hydrogels: A 1372 review. Mater. Sci. Eng. C 2017, 70, 842-855.

1373 (77) Guo, H.; Mussault, C.; Brûlet, A.; Marcellan, A.; Hourdet, D.; Sanson, N. 1374 Thermoresponsive Toughening in LCST-Type Hydrogels with Opposite Topology: From 1375 Structure to Fracture Properties. Macromolecules 2016, 49, 4295-4306.

1376 (78) Guo, H.; Sanson, N.; Marcellan, A.; Hourdet, D. Thermoresponsive Toughening in 1377 LCST-Type Hydrogels: Comparison between Semi-Interpenetrated and Grafted Networks. 1378 Macromolecules 2016, 49, 9568-9577.

1379 (79) Mussault, C.; Guo, H.; Sanson, N.; Hourdet, D.; Marcellan, A. Effect of responsive 1380 graft length on mechanical toughening and transparency in microphase-separated 1381 hydrogels. Soft Matter 2019, 15, 8653-8666.

1382 (80) Rose, S.; Prevoteau, A.; Elziere, P.; Hourdet, D.; Marcellan, A.; Leibler, L. 1383 Nanoparticle solutions as adhesives for gels and biological tissues. Nature 2014, 505, 38213845.

1385 (81) Ida, S.; Morimura, M.; Kitanaka, H.; Hirokawa, Y.; Kanaoka, S. Swelling and 1386 mechanical properties of thermoresponsive/hydrophilic conetworks with crosslinked 1387 domain structures prepared from various triblock precursors. Polym. Chem. 2019, 10, $1388 \quad 6122-6130$.

1389 (82) Ida, S.; Kitanaka, H.; Ishikawa, T.; Kanaoka, S.; Hirokawa, Y. Swelling properties of 1390 thermoresponsive/hydrophilic co-networks with functional crosslinked domain structures. 1391 Polym. Chem. 2018, 9, 1701-1709.

1392 (83) Ida, S.; Katsurada, A.; Yoshida, R.; Hirokawa, Y. Effect of reaction conditions on 1393 poly( $\mathrm{N}$-isopropylacrylamide) gels synthesized by post-polymerization crosslinking 1394 system. React. Funct. Polym. 2017, 115, 73-80.

1395 (84) Ida, S.; Katsurada, A.; Tsujio, M.; Nakamura, M.; Hirokawa, Y. Crosslinker-Based 1396 Regulation of Swelling Behavior of Poly(N-isopropylacrylamide) Gels in a Post1397 Polymerization Crosslinking System. Gels 2019, 6, 2.

1398 (85) Morimura, M.; Ida, S.; Oyama, M.; Takeshita, H.; Kanaoka, S. Design of Hydrogels 1399 with Thermoresponsive Crosslinked Domain Structures via the Polymerization-Induced 1400 Self-Assembly Process and Their Thermoresponsive Toughening in Air. Macromolecules 14012021.

1402 (86) Wang, X.; Qiu, X.; Wu, C. Comparison of the coil-to-globule and the globule-to-coil 1403 transitions of a single poly ( $\mathrm{N}$-isopropylacrylamide) homopolymer chain in water. 1404 Macromolecules 1998, 31, 2972-2976.

1405 (87) Guo, H.; Sanson, N.; Hourdet, D.; Marcellan, A. Thermoresponsive Toughening with 1406 Crack Bifurcation in Phase-Separated Hydrogels under Isochoric Conditions. Adv. Mater. 
(88) Kureha, T.; Hayashi, K.; Li, X.; Shibayama, M. Mechanical properties of temperatureresponsive gels containing ethylene glycol in their side chains. Soft Matter 2020, 16, 10946-10953.

(89) Seuring, J.; Agarwal, S. Polymers with upper critical solution temperature in aqueous solution. Macromol. Rapid Commun. 2012, 33, 1898-920.

(90) Wang, K.; Liu, Q.; Liu, G.; Zeng, Y. Novel thermoresponsive homopolymers of poly[oligo(ethylene glycol) (acyloxy) methacrylate]s: LCST-type transition in water and UCST-type transition in alcohols. Polymer 2020, 203, 122746.

(91) Haas, H. C.; Moreau, R. D.; Schuler, N. W. Synthetic thermally reversible gel systems. II. Journal of Polymer Science Part A - 2: Polym. Phys. 1967, 5, 915-927.

(92) Dai, X.; Zhang, Y.; Gao, L.; Bai, T.; Wang, W.; Cui, Y.; Liu, W. A Mechanically Strong, Highly Stable, Thermoplastic, and Self-Healable Supramolecular Polymer Hydrogel. Adv. Mater. 2015, 27, 3566-3571.

(93) Guo, H.; Mussault, C.; Marcellan, A.; Hourdet, D.; Sanson, N. Hydrogels with Dual Thermoresponsive Mechanical Performance. Macromol. Rapid Commun. 2017, 38, 1700287.

(94) Ning, J.; Li, G.; Haraguchi, K. Synthesis of Highly Stretchable, Mechanically Tough, Zwitterionic Sulfobetaine Nanocomposite Gels with Controlled Thermosensitivities. Macromolecules 2013, 46, 5317-5328.

(95) Ren, Y.; Zhang, Y.; Sun, W.; Gao, F.; Fu, W.; Wu, P.; Liu, W. Methyl matters: An autonomic rapid self-healing supramolecular poly(N-methacryloyl glycinamide) hydrogel. Polymer 2017, 126, 1-8.

(96) Ge, S. J.; Li, J. J.; Geng, J.; Liu, S. N.; Xu, H.; Gu, Z. Z. Adjustable dual temperaturesensitive hydrogel based on a self-assembly cross-linking strategy with highly stretchable and healable properties. Mater. Horizons 2021, 8, 1189-1198.

(97) Chen, F.; Chen, Q.; Zhu, L.; Tang, Z.; Li, Q.; Qin, G.; Yang, J.; Zhang, Y.; Ren, B.; Zheng, J. General Strategy To Fabricate Strong and Tough Low-Molecular-Weight GelatorBased Supramolecular Hydrogels with Double Network Structure. Chem. Mater. 2018, 30, 1743-1754.

(98) Chen, Q.; Zhu, L.; Chen, H.; Yan, H.; Huang, L.; Yang, J.; Zheng, J. A Novel Design Strategy for Fully Physically Linked Double Network Hydrogels with Tough, Fatigue Resistant, and Self-Healing Properties. Adv. Funct. Mater. 2015, 25, 1598-1607.

(99) Chen, Q.; Zhu, L.; Zhao, C.; Wang, Q.; Zheng, J. A Robust, One-Pot Synthesis of Highly Mechanical and Recoverable Double Network Hydrogels Using Thermoreversible Sol-Gel Polysaccharide. Adv. Mater. 2013, 25, 4171-4176.

(100) Van Hoorick, J.; Gruber, P.; Markovic, M.; Tromayer, M.; Van Erps, J.; Thienpont, H.; Liska, R.; Ovsianikov, A.; Dubruel, P.; Van Vlierberghe, S. Cross-Linkable Gelatins with Superior Mechanical Properties Through Carboxylic Acid Modification: Increasing 
the Two-Photon Polymerization Potential. Biomacromolecules 2017, 18, 3260-3272.

1447 (101) Mallick, P. K. 5 - Thermoplastics and thermoplastic-matrix composites for

1448

1449

1450

1451

1452

1453

1454

1455

1456

1457

1458

1459

1460

1461

1462

1463

1464

1465

1466

1467

1468

1469

1470

1471

1472

1473

1474

1475

1476

1477

1478

1479

1480

1481

1482

1483

1484

lightweight automotive structures. In Materials, Design and Manufacturing for Lightweight Vehicles; Mallick, P. K., Ed; Woodhead Publishing: 2010; pp 174-207.

(102) Wang, Y. J.; Zhang, X. N.; Song, Y.; Zhao, Y.; Chen, L.; Su, F.; Li, L.; Wu, Z. L.; Zheng, Q. Ultrastiff and Tough Supramolecular Hydrogels with a Dense and Robust Hydrogen Bond Network. Chem. Mater. 2019, 31, 1430-1440.

(103) Zhang, X. N.; Du, C.; Du, M.; Zheng, Q.; Wu, Z. L. Kinetic insights into glassy hydrogels with hydrogen bond complexes as the cross-links. Materials Today Physics 2020, 15,100230 .

(104) Jiao, C.; Chen, Y.; Liu, T.; Peng, X.; Zhao, Y.; Zhang, J.; Wu, Y.; Wang, H. Rigid and Strong Thermoresponsive Shape Memory Hydrogels Transformed from Poly(vinylpyrrolidone-co-acryloxy acetophenone) Organogels. ACS Appl. Mater. Interfaces 2018, 10, 32707-32716.

(105) Mredha, M. T. I.; Pathak, S. K.; Tran, V. T.; Cui, J.; Jeon, I. Hydrogels with Superior Mechanical Properties from the Synergistic Effect in Hydrophobic-Hydrophilic Copolymers. Chem. Eng. J. 2019, 362, 325-338.

(106) Wang, F.; Weiss, R. A. Thermoresponsive Supramolecular Hydrogels with High Fracture Toughness. Macromolecules 2018, 51, 7386-7395.

(107) Luo, F.; Sun, T. L.; Nakajima, T.; Kurokawa, T.; Li, X.; Guo, H.; Huang, Y.; Zhang, H.; Gong, J. P. Tough Polyion-Complex Hydrogels from Soft to Stiff Controlled by Monomer Structure. Polymer 2017, 116, 487-497.

(108) Ihsan, A. B.; Sun, T. L.; Kurokawa, T.; Karobi, S. N.; Nakajima, T.; Nonoyama, T.; Roy, C. K.; Luo, F.; Gong, J. P. Self-Healing Behaviors of Tough Polyampholyte Hydrogels. Macromolecules 2016, 49, 4245-4252.

(109) Luo, F.; Sun, T. L.; Nakajima, T.; King, D. R.; Kurokawa, T.; Zhao, Y.; Ihsan, A. B.; Li, X.; Guo, H.; Gong, J. P. Strong and Tough Polyion-Complex Hydrogels from Oppositely Charged Polyelectrolytes: A Comparative Study with Polyampholyte Hydrogels. Macromolecules 2016, 49, 2750-2760.

(110) Liu, B.; Xu, Z.; Gao, H.; Fan, C.; Ma, G.; Zhang, D.; Xiao, M.; Zhang, B.; Yang, Y.; Cui, C.; Wu, T.; Feng, X.; Liu, W. Stiffness Self-Tuned Shape Memory Hydrogels for Embolization of Aneurysm. Adv. Funct. Mater. 2020, 30, 1910197.

(111) Berthier, L.; Biroli, G. Theoretical perspective on the glass transition and amorphous materials. Rev.Mod. Phys. 2011, 83, 587-645.

(112) Liang, R.; Yu, H.; Wang, L.; Lin, L.; Wang, N.; Naveed, K. U. Highly Tough Hydrogels with the Body Temperature-Responsive Shape Memory Effect. ACS Appl. Mater. Interfaces 2019, 11, 43563-43572.

(113) Matsuda, A.; Sato, J. i.; Yasunaga, H.; Osada, Y. Order-Disorder Transition of a Hydrogel Containing an n-Alkyl Acrylate. Macromolecules 1994, 27, 7695-7698. 
1485

1486

1487

1488

1489

1490

1491

1492

1493

1494

1495

1496

1497

1498

1499

1500

1501

1502

1503

1504

1505

1506

1507

1508

1509

1510

1511

1512

1513

1514

1515

1516

1517

1518

1519

1520

1521

1522
(114) Osada, Y.; Matsuda, A. Shape memory in hydrogels. Nature 1995, 376, 219.

(115) Bilici, C.; Can, V.; Nöchel, U.; Behl, M.; Lendlein, A.; Okay, O. Melt-Processable Shape-Memory Hydrogels with Self-Healing Ability of High Mechanical Strength. Macromolecules 2016, 49, 7442-7449.

(116) Zhang, K.; Zhao, Z.; Huang, J.; Zhao, T.; Fang, R.; Liu, M. Self-recoverable semicrystalline hydrogels with thermomechanics and shape memory performance. Sci. China Mater. 2018, 62, 586-596.

(117) Tuncaboylu, D. C.; Argun, A.; Sahin, M.; Sari, M.; Okay, O. Structure optimization of self-healing hydrogels formed via hydrophobic interactions. Polymer 2012, 53, 55135522.

(118) Kurt, B.; Gulyuz, U.; Demir, D. D.; Okay, O. High-strength semi-crystalline hydrogels with self-healing and shape memory functions. Eur. Polym. J. 2016, 81, 12-23.

(119) Bilici, C.; Okay, O. Shape Memory Hydrogels via Micellar Copolymerization of Acrylic Acid and n-Octadecyl Acrylate in Aqueous Media. Macromolecules 2013, 46, 3125-3131.

(120) Miyazaki, T.; Kaneko, T.; Gong, J. P.; Osada, Y.; Demura, M.; Suzuki, M. WaterInduced Crystallization of Hydrogels. Langmuir 2002, 18, 965-967.

(121) Okay, O. Semicrystalline physical hydrogels with shape-memory and self-healing properties. J. Mater. Chem. B 2019, 7, 1581-1596.

(122) ACS Appl Mater InterfacesZhao, Z.; Liu, Y.; Zhang, K.; Zhuo, S.; Fang, R.; Zhang, J.; Jiang, L.; Liu, M. Biphasic Synergistic Gel Materials with Switchable Mechanics and Self-Healing Capacity. Angew. Chem. Int. Ed. 2017, 56, 13464-13469.

(123) Zhao, Z.; Zhang, K.; Liu, Y.; Zhou, J.; Liu, M. Highly Stretchable, Shape Memory Organohydrogels Using Phase-Transition Microinclusions. Adv. Mater. 2017, 29, 1701695.

(124) Zhao, Z.; Zhuo, S.; Fang, R.; Zhang, L.; Zhou, X.; Xu, Y.; Zhang, J.; Dong, Z.; Jiang, L.; Liu, M. Dual-Programmable Shape-Morphing and Self-Healing Organohydrogels Through Orthogonal Supramolecular Heteronetworks. Adv. Mater. 2018, 30, 1804435.

(125) Li, L.; Scheiger, J. M.; Levkin, P. A. Design and Applications of Photoresponsive Hydrogels. Adv. Mater. 2019, 31, 1807333.

(126) Tao, Z.; Fan, H.; Huang, J.; Sun, T.; Kurokawa, T.; Gong, J. P. Fabrication of Tough Hydrogel Composites from Photoresponsive Polymers to Show Double-Network Effect. ACS Appl. Mater. Interfaces 2019, 11, 37139-37146.

(127) Moorthy, J. N.; Venkatesan, K.; Weiss, R. G. Photodimerization of Coumarins in Solid Cyclodextrin Inclusion Complexes. J. Org. Chem. 1992, 57, 3292-3297.

(128) Ma, Y.; Hua, M.; Wu, S.; Du, Y.; Pei, X.; Zhu, X.; Zhou, F.; He, X. Bioinspired highpower-density strong contractile hydrogel by programmable elastic recoil. $S c i$. $A d v$. 2020, 6 , eabd2520.

(129) Gao, Y.; Wu, K.; Suo, Z. Photodetachable Adhesion. Adv. Mater. 2019, 31, 1806948. 
(130) Guvendiren, M.; Burdick, J. A. Stiffening hydrogels to probe short- and long-term cellular responses to dynamic mechanics. Nat. Commun. 2012, 3, 792.

(131) Rowlands, A. S.; George, P. A.; Cooper-White, J. J. Directing osteogenic and myogenic differentiation of MSCs: interplay of stiffness and adhesive ligand presentation. Am. J. Physiol., Cell Physiol. 2008, 295, C1037-C1044.

(132) Lee, I. N.; Dobre, O.; Richards, D.; Ballestrem, C.; Curran, J. M.; Hunt, J. A.; Richardson, S. M.; Swift, J.; Wong, L. S. Photoresponsive Hydrogels with Photoswitchable Mechanical Properties Allow Time-Resolved Analysis of Cellular Responses to Matrix Stiffening. ACS Appl. Mater. Interfaces 2018, 10, 7765-7776.

(133) Rosales, A. M.; Mabry, K. M.; Nehls, E. M.; Anseth, K. S. Photoresponsive Elastic Properties of Azobenzene-Containing Poly(ethylene-glycol)-Based Hydrogels. Biomacromolecules 2015, 16, 798-806.

(134) Yang, X.; Liu, X.; Liu, Z.; Pu, F.; Ren, J.; Qu, X. Near-infrared light-triggered, targeted drug delivery to cancer cells by aptamer gated nanovehicles. Adv. Mater. 2012, 24, 2890-2895.

(135) Fujigaya, T.; Morimoto, T.; Niidome, Y.; Nakashima, N. NIR Laser-Driven Reversible Volume Phase Transition of Single-Walled Carbon Nanotube/Poly(Nisopropylacrylamide) Composite Gels. Adv. Mater. 2008, 20, 3610-3614.

(136) Zhu, C. H.; Lu, Y.; Chen, J. F.; Yu, S. H. Photothermal poly(Nisopropylacrylamide)/Fe3O4 nanocomposite hydrogel as a movable position heating source under remote control. Small 2014, 10, 2796-2800.

(137) Li, W.; Wang, J.; Ren, J.; Qu, X. 3D Graphene Oxide-Polymer Hydrogel: NearInfrared Light-Triggered Active Scaffold for Reversible Cell Capture and On-Demand Release. Adv. Mater. 2013, 25, 6737-6743.

(138) Huang, J.; Zhao, L.; Wang, T.; Sun, W.; Tong, Z. NIR-Triggered Rapid Shape Memory PAM-GO-Gelatin Hydrogels with High Mechanical Strength. ACS Appl. Mater. Interfaces 2016, 8, 12384-12392.

(139) Dai, C. F.; Zhang, X. N.; Du, C.; Frank, A.; Schmidt, H. W.; Zheng, Q.; Wu, Z. L. Photoregulated Gradient Structure and Programmable Mechanical Performances of Tough Hydrogels with a Hydrogen-Bond Network. ACS Appl. Mater. Interfaces 2020, 12, 5337653384.

(140) Dai, C. F.; Du, C.; Xue, Y.; Zhang, X. N.; Zheng, S. Y.; Liu, K.; Wu, Z. L.; Zheng, Q. Photodirected Morphing Structures of Nanocomposite Shape Memory Hydrogel with High Stiffness and Toughness. ACS Appl. Mater. Interfaces 2019, 11, 43631-43640.

(141) Ryplida, B.; Lee, K. D.; In, I.; Park, S. Y. Light - Induced Swelling - Responsive Conductive, Adhesive, and Stretchable Wireless Film Hydrogel as Electronic Artificial Skin. Adv. Funct. Mater. 2019, 29, 1903209.

(142) Hua, L.; Xie, M.; Jian, Y.; Wu, B.; Chen, C.; Zhao, C. Multiple-Responsive and Amphibious Hydrogel Actuator Based on Asymmetric UCST-Type Volume Phase 
1563 (143) Scheiger, J. M.; Levkin, P. A. Hydrogels with Preprogrammable Lifetime via UV1564 Induced Polymerization and Degradation. Adv. Funct. Mater. 2020, 30, 1909800.

1565 (144) Koetting, M. C.; Peters, J. T.; Steichen, S. D.; Peppas, N. A. Stimulus-responsive 1566 hydrogels: Theory, modern advances, and applications. Mater. Sci. Eng. R Rep. 2015, 93, $1567 \quad 1-49$.

1568 (145) Steiner, T. The Hydrogen Bond in the Solid State. Angew. Chem. Int. Ed. 2002, 41, $156948-76$.

1570 (146) Gao, F.; Zhang, Y.; Li, Y.; Xu, B.; Cao, Z.; Liu, W. Sea Cucumber-Inspired Autolytic 1571 Hydrogels Exhibiting Tunable High Mechanical Performances, Repairability, and 1572 Reusability. ACS Appl. Mater. Interfaces 2016, 8, 8956-66.

1573 (147) Wang, Y. J.; Li, C. Y.; Wang, Z. J.; Zhao, Y.; Chen, L.; Wu, Z. L.; Zheng, Q. Hydrogen 1574 bond-reinforced double-network hydrogels with ultrahigh elastic modulus and shape 1575 memory property. J. Polym. Sci., Part B: Polym. Phys. 2018, 56, 1281-1286.

(148) Ding, H.; Zhang, X. N.; Zheng, S. Y.; Song, Y.; Wu, Z. L.; Zheng, Q. Hydrogen bond reinforced poly(1-vinylimidazole-co-acrylic acid) hydrogels with high toughness, fast selfrecovery, and dual pH-responsiveness. Polymer 2017, 131, 95-103.

(149) Zhang, X. N.; Wang, Y. J.; Sun, S.; Hou, L.; Wu, P.; Wu, Z. L.; Zheng, Q. A Tough and Stiff Hydrogel with Tunable Water Content and Mechanical Properties Based on the Synergistic Effect of Hydrogen Bonding and Hydrophobic Interaction. Macromolecules 2018, 51, 8136-8146.

(150) Li, X.; Li, R.; Liu, Z.; Gao, X.; Long, S.; Zhang, G. Integrated Functional HighStrength Hydrogels with Metal-Coordination Complexes and H-Bonding Dual Physically Cross-linked Networks. Macromol. Rapid Commun. 2018, 39, 1800400.

(151) Yu, H. C.; Li, C. Y.; Du, M.; Song, Y.; Wu, Z. L.; Zheng, Q. Improved Toughness and Stability of $\kappa$-Carrageenan/Polyacrylamide Double-Network Hydrogels by Dual CrossLinking of the First Network. Macromolecules 2019, 52, 629-638.

(152) Das Mahapatra, R.; Imani, K. B. C.; Yoon, J. Integration of Macro-Cross-Linker and Metal Coordination: A Super Stretchable Hydrogel with High Toughness. ACS Appl. Mater. Interfaces 2020, 12, 40786-40793.

1595 (154) Henderson, K. J.; Zhou, T. C.; Otim, K. J.; Shull, K. R. Ionically Cross-Linked 1596 Triblock Copolymer Hydrogels with High Strength. Macromolecules 2010, 43, 6193-6201.

1597 (155) Nisato, G.; Skouri, R.; Schosseler, F.; Munch, J.-P.; Candau, S. J. Elastic behaviour 1598 of salt-free polyelectrolyte gels. Faraday Discuss. 1995, 101, 133-146.

1599 (156) Xiao, Y. Y.; Gong, X. L.; Kang, Y.; Jiang, Z. C.; Zhang, S.; Li, B. J. Light-, pH- and 
1600 thermal-responsive hydrogels with the triple-shape memory effect. Chem. Commun. 2016, $160152,10609-10612$.

1602 (157) Li, Y.; Tanaka, T. Kinetics of swelling and shrinking of gels. J. Chem. Phys. 1990, 1603 92, 1365-1371.

1604 (158) Pearson, R. G. Hard and Soft Acids and Bases. In Survey of Progress in Chemistry; 1605 Scott, A. F., Ed.; Elsevier: 1969; pp 1-52.

1606 (159) Chen, Q.; Yan, X.; Zhu, L.; Chen, H.; Jiang, B.; Wei, D.; Huang, L.; Yang, J.; Liu, 1607 B.; Zheng, J. Improvement of Mechanical Strength and Fatigue Resistance of Double 1608 Network Hydrogels by Ionic Coordination Interactions. Chem. Mater. 2016, 28, 5710-5720.

1609 (160) Sun, J. Y.; Zhao, X.; Illeperuma, W. R.; Chaudhuri, O.; Oh, K. H.; Mooney, D. J.; 1610 Vlassak, J. J.; Suo, Z. Highly stretchable and tough hydrogels. Nature 2012, 489, 133-136.

1611 (161) Lane, D. D.; Kaur, S.; Weerasakare, G. M.; Stewart, R. J. Toughened hydrogels 1612 inspired by aquatic caddisworm silk. Soft Matter 2015, 11, 6981-90.

1613 (162) Hu, Y.; Du, Z.; Deng, X.; Wang, T.; Yang, Z.; Zhou, W.; Wang, C. Dual Physically 1614 Cross-Linked Hydrogels with High Stretchability, Toughness, and Good Self1615 Recoverability. Macromolecules 2016, 49, 5660-5668.

1616 (163) Cao, J.; Wang, Y.; He, C.; Kang, Y.; Zhou, J. Ionically crosslinked 1617 chitosan/poly(acrylic acid) hydrogels with high strength, toughness and antifreezing 1618 capability. Carbohydr. Polym. 2020, 242, 116420.

1619 (164) Li, J.; Illeperuma, W. R. K.; Suo, Z.; Vlassak, J. J. Hybrid Hydrogels with Extremely 1620 High Stiffness and Toughness. ACS Macro Lett. 2014, 3, 520-523.

1621 (165) Yu, H. C.; Zheng, S. Y.; Fang, L.; Ying, Z.; Du, M.; Wang, J.; Ren, K.-F.; Wu, Z. L.; 1622 1623

1624 Zheng, Q. Reversibly Transforming a Highly Swollen Polyelectrolyte Hydrogel to an Extremely Tough One and its Application as a Tubular Grasper. Adv. Mater. 2020, 32, 2005171.

1625 (166) Baldwin, R. L. How Hofmeister ion interactions affect protein stability. Biophys. J. 1626 1996, 71, 2056-2063.

1627 (167) Jungwirth, P.; Cremer, P. S. Beyond Hofmeister. Nat. Chem. 2014, 6, 261-263.

1628 (168) Zhang, Y.; Cremer, P. S. Chemistry of Hofmeister anions and osmolytes. Annu. Rev. 1629 Phys. Chem. 2010, 61, 63-83.

1630 (169) Zhang, Y.; Furyk, S.; Bergbreiter, D. E.; Cremer, P. S. Specific Ion Effects on the 1631 Water Solubility of Macromolecules: PNIPAM and the Hofmeister Series. J. Am. Chem. 1632 Soc. 2005, 127, 14505-14510.

1633 (170) Tuncaboylu, D. C.; Sari, M.; Oppermann, W.; Okay, O. Tough and Self-Healing 1634 Hydrogels Formed via Hydrophobic Interactions. Macromolecules 2011, 44, 4997-5005.

1635 (171) Sun, X.; Luo, C.; Luo, F. Preparation and properties of self-healable and conductive 1636 PVA-agar hydrogel with ultra-high mechanical strength. Eur. Polym. J. 2020, 124, 109465. 
1637 (172) Chen, W.; Li, D.; Bu, Y.; Chen, G.; Wan, X.; Li, N. Design of strong and tough 1638 methylcellulose-based hydrogels using kosmotropic Hofmeister salts. Cellulose 2019, 27, 1639 1113-1126.

1640 (173) Wu, S.; Hua, M.; Alsaid, Y.; Du, Y.; Ma, Y.; Zhao, Y.; Lo, C. Y.; Wang, C.; Wu, D.; 1641 Yao, B.; Strzalka, J.; Zhou, H.; Zhu, X.; He, X. Poly(vinyl alcohol) Hydrogels with Broad1642 Range Tunable Mechanical Properties via the Hofmeister Effect. Adv. Mater. 2021, 1643 e2007829.

1644 (174) Li, P.; Wang, Z.; Lin, X.; Wang, X.; Guo, H. Muscle-Inspired Ion-Sensitive 1645 Hydrogels with Highly Tunable Mechanical Performance for Versatile Engineering 1646 Applications. Sci. China Mater. 2021.

1647 (175) Cui, C.; Shao, C.; Meng, L.; Yang, J. High-Strength, Self-Adhesive, and Strain1648 Sensitive Chitosan/Poly(acrylic acid) Double-Network Nanocomposite Hydrogels 1649 Fabricated by Salt-Soaking Strategy for Flexible Sensors. ACS Appl. Mater. Interfaces 1650 2019, 11, 39228-39237.

1651 (176) Sun, T. L.; Kurokawa, T.; Kuroda, S.; Ihsan, A. B.; Akasaki, T.; Sato, K.; Haque, M. 1652 A.; Nakajima, T.; Gong, J. P. Physical Hydrogels Composed of Polyampholytes 1653 Demonstrate High Toughness and Viscoelasticity. Nat. Mater. 2013, 12, 932-937.

1654 (177) Luo, F.; Sun, T. L.; Nakajima, T.; Kurokawa, T.; Zhao, Y.; Sato, K.; Ihsan, A. B.; Li, 1655 X.; Guo, H.; Gong, J. P. Oppositely charged polyelectrolytes form tough, self-healing, and 1656 rebuildable hydrogels. Adv. Mater. 2015, 27, 2722-2727.

1657 (178) Fan, H.; Wang, J.; Tao, Z.; Huang, J.; Rao, P.; Kurokawa, T.; Gong, J. P. Adjacent 1658 cationic-aromatic sequences yield strong electrostatic adhesion of hydrogels in seawater. 1659 Nat. Commun. 2019, 10, 5127.

1660 (179) Means, A. K.; Grunlan, M. A. Modern Strategies To Achieve Tissue-Mimetic, 1661 Mechanically Robust Hydrogels. ACS Macro Lett. 2019, 8, 705-713. 